\title{
QUARK REGGE TRAJECTORY IN TWO LOOPS FROM UNITARITY RELATIONS
}

\author{
A. V. Bogdan ${ }^{1, *}$ and V. S. Fadin ${ }^{1, \dagger}$ \\ ${ }^{1}$ Institute for Nuclear Physics, 630090 Novosibirsk, Russia \\ and Novosibirsk State University, 630090 Novosibirsk, Russia
}

\begin{abstract}
The two-loop quark Regge trajectory is obtained at arbitrary space-time dimension $D$ using the $s$-channel unitarity conditions. Although explicit calculations are performed for massless quarks, the method used allows to find the trajectory for massive quarks as well. At $D \rightarrow 4$ the trajectory turns into one derived earlier from the high-energy limit of the two-loop amplitude for the quark-gluon scattering. The comparison of two expressions, obtained by quite different methods, serves as a strict cross check of many intermediate results used in the calculations, and their agreement gives a strong evidence of accuracy of these results.
\end{abstract}

\section{INTRODUCTION}

Perturbative QCD is widely used for the description of semihard [1] as well as hard processes [2]. But whereas the theory of the latter ones is clear and plain, a lot of problems remains unsolved for the former processes. The applicability of perturbation theory, improved by the renormalization group, to a hard process with a large typical virtuality $Q^{2}$ is justified by the smallness of the strong coupling constant $\alpha_{s}\left(Q^{2}\right)$. In the semihard case, however, smallness of the ratio $x$ of the typical virtuality $Q^{2}$ to the squared c.m.s. energy $s$ requires resummation of the terms strengthened by powers of $\ln (1 / x)$. In the scattering channel this problem is related to the theoretical description of high energy amplitudes at fixed (not growing with $s$ ) momentum transfer $t$. It turns out, that the Gribov-Regge theory of complex angular momenta, which was developed much before appearance of QCD, is eminently suitable for description of the QCD amplitudes, due to the remarkable property of QCD - Reggeization of its elementary particles, gluons and quarks [3]-[6]. The Reggeization means, in particular, that with account of radiative corrections in the high energy limit $s$-dependence of QCD amplitudes with gluon $(G)$ or quark $(Q)$ quantum numbers in the $t$-channel is given by Regge factors $(s)^{j_{P}(t)}$, with $P=G$ or $P=Q$ accordingly. The functions $j_{P}(t)$ with the property $j_{P}\left(m_{P}^{2}\right)=s_{P}$ ( $m_{P}$ and $s_{P}$ are respective mass and spin values), called Regge trajectory, describe motion of poles of corresponding $t$-channel partial waves in the complex angular momentum plane. In this respect QCD sharply differs from QED, where only amplitudes with electron exchange in the $t$-channel [7], but not with photon one [8], acquire the Regge factors.

The Reggeization phenomenon is extremely important at high energy. The gluon Reggeization is especially significant, since gluon exchanges in the $t$-channel provide nondecreasing at large $s$ cross sections. In particular, the gluon Reggeization constitutes the basis of the famous BFKL approach [5, 9] to the theoretical description of high energy pro-

*Electronic address: A.B.Bogdan@inp.nsk.su

${ }^{\dagger}$ Electronic address: Fadin@inp.nsk.su 
cesses in QCD. Formulated originally in leading logarithmic approximation (LLA), the BFKL approach is developed now in next-to-leading one (for references see, for instance, [10]), since LLA is not sufficiently reliable, especially because it does not fix scales neither longitudinal (for $s$ ), nor transverse (for running coupling $\alpha_{s}$ ) momenta. This development extensively uses the gluon Reggeization, which has been proved in LLA [11], but in next-to-leading approximation (NLA) till now remains a hypothesis, although successfully passed through a set of stringent tests on self-consistency (see, for instance, [12] and references therein). Accordingly, the next-to-leading order (NLO) gluon Regge trajectory and Reggeized gluon vertices are calculated; moreover, a way for the proof of the gluon Reggeization in NLA is outlined (see, e.g., [10] and references therein).

Along with the Pomeron, which appears in the BFKL approach as a compound state of two Reggeized gluons, the hadron phenomenology requires Reggeons, which can be constructed in QCD as colorless states of Reggeized quarks and antiquarks. It demands further development of the Reggeized quark theory which remains in a worse state than the Reggeized gluon theory, although a noticeable progress was achieved last years. In particular, multi-particle Reggeon vertices required in NLA were found [13] and the NLO corrections to the LLA vertices were calculated $[14,15]$ assuming the quark Reggeization in NLA. Note that the Reggeization hypothesis is extremely powerful; but in the quark case actually it was not proved even in LLA, where merely its self-consistency was shown, in all orders of $\alpha_{s}$ but only in a particular case of elastic quark-gluon scattering [6]. Recently the hypothesis was tested at NLO in order $\alpha_{s}^{2}$ in [16], where its compatibility with the two-loop amplitude for the quark-gluon scattering was exhibited and the NLO correction to the quark trajectory was found in the limit of the space-time dimension $D$ tending to the physical value $D=4$.

In this paper we investigate the quark Reggeization in NLO by the method based on the $s$-channel unitarity and the analyticity of scattering amplitudes, which was developed for analysis of processes with gluon exchanges $[4,5]$ and was already successfully applied to processes with fermion exchanges [6]. The two-loop quark trajectory at arbitrary space-time dimension $D$ is obtained as a particular result of the investigation. At $D \rightarrow 4$ the trajectory goes into one derived in [16]. This agreement testifies to accuracy of many intermediate results used in both derivations. In the method used here the trajectory is obtained from the requirement of the compatibility of the Reggeized form of the amplitudes with the $s$ channel unitarity at the two-loop level. A possible generalization of this requirement to all orders of perturbation theory should give the "bootstrap" conditions on the Reggeized quark vertices and the trajectory in QCD. Verification of them will give a strict test for the quark Reggeization. A proof of the Reggeization is also possible on this way.

The calculation of the two-loop corrections to the quark trajectory is performed explicitly for massless quarks; but the method used here allows to do it for massive quarks as well, since all necessary one-loop Reggeon vertices for the massive case are known now.

The paper is organized as follows. In the next Section all necessary denotations are introduced and the method of calculation is discussed. Section 3 is devoted to the calculation of the two-particle contribution to the $s$-channel discontinuity of the quark-gluon scattering amplitude. The contribution of the three-particle intermediate state is calculated in Section 4. The final expressions for the discontinuity and the two-loop corrections to the quark trajectory are presented and discussed in Section 5. For convenience, the integrals encountered in Sections 3 and 4 are listed in Appendices $\mathbf{A}$ and $\mathbf{B}$ respectively. Details of the calculation of a new nontrivial integral arising in present calculations are given in Appendix $\mathbf{C}$. 


\section{DENOTATIONS AND METHOD OF CALCULATION}

Let us consider the backward quark-gluon scattering process (see Fig. 1) in the limit of large (tending to infinity) c.m.s. energy and fixed momentum transfer $t \equiv q^{2}=\left(p_{Q}-p_{G^{\prime}}\right)^{2}$.

We use the Sudakov decomposition of momenta

$$
\begin{gathered}
p=\beta p_{1}+\alpha p_{2}+p_{\perp}, \\
p_{1}^{2}=p_{2}^{2}=0, \quad\left(p_{1}+p_{2}\right)^{2}=s, \quad s \alpha \beta=p^{2}-p_{\perp}^{2},
\end{gathered}
$$

supposing that the momenta $p_{G}, p_{Q^{\prime}}$ and $p_{Q}, p_{G^{\prime}}$ are close to the light-cone momenta $p_{1}$ and $p_{2}$ respectively, that is

$$
\beta_{G} \sim \beta_{Q^{\prime}} \sim \alpha_{Q} \sim \alpha_{G^{\prime}} \simeq 1, \quad \beta_{Q} \simeq \beta_{G^{\prime}} \simeq \alpha_{G} \simeq \alpha_{Q^{\prime}} \sim \frac{|t|}{s},
$$

and all transverse momenta are limited, so that $q \simeq q_{\perp}$. We don't suppose that $p_{1}$ and $p_{2}$ are contained in the initial momentum plane, in order to maintain symmetry between cross channels and to make more evident substitutions for transitions between channels. For a gluon having momentum $k_{a}\left(k_{b}\right)$ with predominant component along $p_{1}\left(p_{2}\right)$ we use physical polarization vectors in the light-cone gauge $e\left(k_{a}\right) p_{2}=0\left(e\left(k_{b}\right) p_{1}=0\right)$, so that

$$
e\left(k_{a}\right)=e\left(k_{a}\right)_{\perp}-\frac{\left(e\left(k_{a}\right) k_{a}\right)_{\perp}}{k_{a} p_{2}} p_{2}, e\left(k_{b}\right)=e\left(k_{b}\right)_{\perp}-\frac{\left(e\left(k_{b}\right) k_{b}\right)_{\perp}}{k_{b} p_{1}} p_{1},
$$

where $(a b)_{\perp}$ means $\left(a_{\perp} b_{\perp}\right)$. Furthermore, since with our choice of gauges gluon polarization vectors are expressed in terms of their transverse components, from now we will use only these components, without explicit indications, so that everywhere below $e$ means $e_{\perp}$. The same we will do for the momentum transfer $q$.

The large $s$ and fixed $t$ limit of scattering amplitudes is related to quantum numbers in the $t$-channel. For the gauge group $S U\left(N_{c}\right) t$-channel colour state of the process depicted at Fig. 1 contains three irreducible representations of the colour group (for QCD with $N_{c}=3$ it is $\underline{3} \oplus \underline{\overline{6}} \oplus \underline{15})$. Therefore it is natural to decompose the quark-gluon scattering amplitude into three parts, in accordance with the representations $[6,16]$. At the same time, in the GribovRegge theory each part must be decomposed into two pieces according to the new quantum number - signature, which is introduced in the theory. Besides quantum numbers, commonly used for particle classification, a Reggeon has definite signature, positive or negative, which is actually a parity of the $t$-channel partial waves in respect of the substitution $\cos \theta_{t} \rightarrow$ $-\cos \theta_{t}$ (that turns into $s \rightarrow u=-s$ in the limit of large $s$ and fixed $t$ ). Consequently, there are six terms $A_{\chi}^{( \pm)}, \quad \chi=\underline{3}, \underline{\overline{6}}$ and $\underline{15}$ in full decomposition $[6,16]$ of the quarkgluon scattering amplitude. As it is known [6], in LLA only one of the positive signature amplitudes, namely $A_{\underline{3}}^{(+)}$, does survive; and at the same time it has the Reggeized form. It is not so for the negative signature amplitudes. The Bethe-Salpiter type equation obtained for them in LLA [6] has not a simple Regge-type solution (in fact, no solution has been found at all). Note that they are not actually leading in each order of perturbation theory, because leading logarithms cancel in them as the result of antisymmetry with respect to the exchange $s \rightarrow u=-s$. Below we consider only the amplitudes of positive signature. As we will see, in NLA, as well as in LLA, only amplitudes with a colour triplet in the $t$-channel survives out of them. For the quark-gluon scattering the contribution of the Reggeized quark can be represented as (here and below we write symbols of initial (final) particles as lower (upper) 
indices of scattering amplitudes and put on the first place particles with momenta close to $\left.p_{1}\right)$ :

$$
\mathcal{R}_{G Q}^{Q^{\prime} G^{\prime}}=\Gamma_{Q^{\prime} G} \frac{1}{m_{Q}-\hat{q}} \frac{1}{2}\left[\left(\frac{-s}{-t}\right)^{\delta_{\mathrm{T}}(\hat{q})}+\left(\frac{s}{-t}\right)^{\delta_{\mathrm{T}}(\hat{q})}\right] \Gamma_{G^{\prime} Q},
$$

where $\Gamma_{Q^{\prime} G}$ and $\Gamma_{G^{\prime} Q}$ are effective vertices for interaction of particles (quarks and gluons) with the Regeized quark; we call them PPR vertices, and we call $\delta_{\mathrm{T}}(\hat{q})$ - the quark trajectory. Strictly speaking, for massive quarks there are two trajectories, in accordance with two possible parity states for a off mass shell quark. These trajectories are determined by eigenvalues of $\delta_{\mathrm{T}}(\hat{q})$. We perform actual calculations for the massless case, when $\delta_{\mathrm{T}}(\hat{q})$ depends in fact not on $\hat{q}$, but on $\hat{q}^{2}=t$, and write it as $\delta_{\mathrm{T}}(t)$. Note, however, that the PPR vertices $\Gamma_{Q^{\prime} G}$ and $\Gamma_{G^{\prime} Q}$ are known now for massive quarks [15], so that all consideration presented below can be transferred on the massive case in a straightforward way.

We demonstrate that the Reggeized form (4) is compatible with the $s$-channel unitarity and obtain the NLO contribution to the trajectory $\delta_{\mathrm{T}}$. More precisely, we calculate, using the unitarity relation, both logarithmic and non-logarithmic terms in the two-loop $s$-channel discontinuity of the backward quark-gluon scattering amplitude with positive signature and prove that only colour triplet $t$-channel states contribute to the discontinuity. It means that only the colour triplet part $A_{\underline{3}}^{(+)}$of the amplitude does survive at NLO as well as at LO. We compare the calculated discontinuity with the discontinuity of the Reggeized quark contribution (4). The logarithmic terms of confronted discontinuities turn out to be equal. The non-logarithmic terms in the discontinuity of (4) are expressed through the oneloop corrections to the PPR vertices, which are known, and the two-loop correction to the trajectory, that makes possible to obtain the last correction.

For massless quarks the PPR vertices entering in (4) have the form [14]:

$$
\begin{aligned}
& \Gamma_{Q^{\prime} G}=-g \bar{u}\left(p_{Q^{\prime}}\right) t^{G}\left[\hat{e}_{G}\left(1+\delta_{e}(t)\right)+\frac{\left(e_{G} q\right) \hat{q}}{q^{2}} \delta_{q}(t)\right], \\
& \Gamma_{G^{\prime} Q}=-g\left[\hat{e}_{G^{\prime}}^{*}\left(1+\delta_{e}(t)\right)+\frac{\left(e_{G^{\prime}}^{*} q\right) \hat{q}}{q^{2}} \delta_{q}(t)\right] t^{G^{\prime}} u\left(p_{Q}\right) .
\end{aligned}
$$

Here $t^{G}, t^{G^{\prime}}$ are the color group generators in the fundamental representation; we omit colour wave functions for gluons and assume that they are included in $u\left(p_{Q}\right), \bar{u}\left(p_{Q^{\prime}}\right)$ for quarks. The one-loop corrections $\delta_{e}(t), \delta_{q}(t)$ can be written as

$$
\delta_{e}(t)=\omega^{(1)}(t) \delta_{e}^{(1)}, \quad \delta_{q}(t)=\omega^{(1)}(t) \delta_{q}^{(1)},
$$

where

$$
\begin{gathered}
\delta_{e}^{(1)}=\frac{C_{F}}{2 N_{c}}\left(\frac{1}{\epsilon}-\frac{3(1-\epsilon)}{2(1+2 \epsilon)}+\psi(1)+\psi(1-\epsilon)-2 \psi(1+\epsilon)\right)+\frac{1}{2 \epsilon}-\frac{\epsilon}{2(1+2 \epsilon)}, \\
\delta_{q}^{(1)}=\frac{\epsilon}{2(1+2 \epsilon)}\left(1+\frac{1}{N_{c}^{2}}\right),
\end{gathered}
$$

$\psi(x)=\Gamma^{\prime}(x) / \Gamma(x)$ is the logarithmic derivative of the Euler gamma function, and $\omega^{(1)}(t)$ is the one-loop gluon Regge trajectory:

$$
\omega^{(1)}(t)=-\frac{2 N_{c}}{\epsilon} \frac{g_{s}^{2}}{(4 \pi)^{2+\epsilon}}\left(\frac{\mu^{2}}{-t}\right)^{-\epsilon} \Gamma_{\epsilon}, \quad \Gamma_{\epsilon}=\frac{\Gamma^{2}(1+\epsilon) \Gamma(1-\epsilon)}{\Gamma(1+2 \epsilon)} .
$$


We use conventional dimensional regularization; the space-time dimension $D=4+2 \epsilon$ and $g_{s}=g \mu^{\epsilon}$ is the dimensionless bare coupling constant.

To avoid uncertainties let us note that the vertices $\Gamma_{G \bar{Q}}$ and $\Gamma_{\bar{Q} G^{\prime}}$ are obtained from (5) by the substitutions $\bar{u}\left(p_{Q^{\prime}}\right) \rightarrow \bar{v}\left(p_{\bar{Q}}\right), \quad e_{G} \rightarrow e_{G}^{*} \quad$ and $u\left(p_{Q}\right) \rightarrow v\left(p_{\bar{Q}}\right), \quad e_{G^{\prime}}^{*} \rightarrow e_{G^{\prime}}$ respectively.

Representing the quark trajectory as

$$
\delta_{\mathrm{T}}(t)=\frac{\omega^{(1)}(t)}{N_{c}} \delta_{\mathrm{T}}^{(1)}+\frac{1}{2}\left(\frac{\omega^{(1)}(t)}{N_{c}}\right)^{2} \delta_{\mathrm{T}}^{(2)}
$$

where $\delta_{\mathrm{T}}^{(1)}=C_{F}[6]$, and the two-loop $s$-channel discontinuity of the Reggeized quark contribution (4) as

$$
\left[\mathcal{R}_{G Q}^{Q^{\prime} G^{\prime}}(\text { two-loop })\right]_{s}=\frac{i \pi g^{2}}{t} \frac{1}{2}\left(\frac{\omega^{(1)}(t)}{N_{c}}\right)^{2} \bar{u}\left(p_{Q^{\prime}}\right) t^{G} t^{G^{\prime}} \Delta_{R} u\left(p_{Q}\right),
$$

we have from (4), (5) (9) and (6)

$$
\Delta_{R}=\delta_{\mathrm{T}}^{(2)} \hat{\mathcal{Q}}+2 C_{F}\left(2 N_{c} \delta_{e}^{(1)}+C_{F} \ln \frac{s}{-t}\right) \hat{\mathcal{Q}}+2 C_{F} N_{c} \delta_{q}^{(1)} \hat{\mathcal{E}}
$$

where

$$
\hat{\mathcal{Q}}=\hat{e}_{G} \hat{q} \hat{e}_{G^{\prime}}^{*}, \quad \hat{\mathcal{E}}=\hat{e}_{G}\left(e_{G^{\prime}}^{*} q\right)+\hat{e}_{G^{\prime}}^{*}\left(e_{G} q\right)
$$

and $\delta_{e}^{(1)}, \delta_{q}^{(1)}$ are given by $(7)$.

Below we calculate the discontinuity $\left[\left(\mathcal{A}^{(+)}\right)_{G Q}^{Q^{\prime} G^{\prime}} \text { (two-loop) }\right]_{s}$ of the backward quarkgluon scattering amplitude with positive signature from the $s$-channel unitarity condition. We show, that it has the same colour structure as $\left[\mathcal{R}_{G Q}^{Q^{\prime} G^{\prime}} \text { (two-loop) }\right]_{s}(10)$, that is in this approximation only the colour triplet state survives in the positive signature. Writing the calculated discontinuity in the same form as the right hand side of (10) with $\Delta_{s}$ instead of $\Delta_{R}$, we see that $\Delta_{s}$ has the same helicity structure as $\Delta_{R}(11)$ and that their logarithmic terms coincide; moreover, the non-logarithmic terms at the helicity non-conserving structure $\hat{\mathcal{E}}$ also do coincide. After that the requirement of equality of the non-logarithmic terms at the helicity conserving structure $\hat{\mathcal{Q}}$ gives us $\delta_{\mathrm{T}}^{(2)}$.

The calculation of $\Delta_{s}$ is the main content of this paper. It is determined from the $s^{-}$ channel unitarity relation:

$$
\begin{aligned}
{\left[\left(\mathcal{A}^{(+)}\right)_{G Q}^{Q^{\prime} G^{\prime}}(\text { two-loop })\right]_{s} } & =\frac{i \pi g^{2}}{t} \frac{1}{2}\left(\frac{\omega^{(1)}(t)}{N_{c}}\right)^{2} \bar{u}\left(p_{Q^{\prime}}\right) t^{G} t^{G^{\prime}} \Delta_{s} u\left(p_{Q}\right) \\
& =i \mathrm{P}^{(+)} \sum_{n} \int d \Phi_{n} \mathcal{A}_{G Q}^{n}\left(\mathcal{A}_{Q^{\prime} G^{\prime}}^{n}\right)^{*}
\end{aligned}
$$

where $\Phi_{n}$ is the $n$-particle phase space element and $\mathrm{P}^{(+)}$is the positive signature projector. The summation is performed over two- and three-particle intermediate states; accordingly, we represent the discontinuity as the sum of two contributions

$$
\Delta_{s}=\Delta_{s}^{(2)}+\Delta_{s}^{(3)}
$$


The projection on positive signature means the half-sum of the $s$-channel discontinuities for the direct $\left(G Q \rightarrow Q^{\prime} G^{\prime}\right)$ and the crossed $\left(G \tilde{G} \rightarrow Q^{\prime} \bar{Q}^{\prime}\right)$ processes. More precisely, if one represents the discontinuity of the direct processes as $\left\langle Q^{\prime} G^{\prime}|M| G Q\right\rangle$, where $|G Q\rangle$ is a spin and colour quark-gluon wave function, and the discontinuity for the process $G \tilde{G} \rightarrow Q^{\prime} \bar{Q}^{\prime}$ with $p_{\tilde{G}}=p_{Q}, p_{\bar{Q}^{\prime}}=p_{G^{\prime}}$ as $\left\langle Q^{\prime} \bar{Q}^{\prime}\left|M_{c}\right| G \tilde{G}\right\rangle$, then the projection on positive signature is $\left\langle Q^{\prime} G^{\prime}\left|\left(M+M_{c}\right) / 2\right| G Q\right\rangle$. Note that calculating $\Delta_{s}$ we always use that rightmost $\hat{p}_{2}$ and leftmost $\hat{p}_{1}$ in $M$ and $M_{c}$ give negligible contributions because of the Dirac equation.

\section{TWO-PARTICLE CONTRIBUTION TO THE DISCONTINUITY}

In the direct channel a two-particle intermediate state can be solely quark-gluon one. Since only limited transverse momenta of intermediate particles are important in the unitarity relation (we will see it directly; actually, it is a consequence of the renormalizability), non-negligible contributions are given by two non-overlapping kinematical regions. In one of them intermediate gluon momentum is close to $p_{G}$ (see Fig. 2a), and in another to $p_{Q}$ (Fig. 2b). In both cases the amplitudes in the right hand side of the unitarity relation (14) are in Regge type kinematics.

As we need to calculate the two-loop contribution to the discontinuity, one of them has to be taken in the Born approximation and another one in the one-loop approximation. An important point is that since Born amplitudes are real, only real parts of one-loop amplitudes are essential for the calculation of the discontinuity. Therefore required amplitudes are determined by Reggeized quark and gluon contributions. Moreover, we can use $\mathcal{A}_{n}^{Q^{\prime} G^{\prime}}$ instead of $\left(\mathcal{A}_{Q^{\prime} G^{\prime}}^{n}\right)^{*}$, as imaginary parts of the amplitudes are not important.

The amplitudes with $t$-channel quarks can be obtained by evident substitutions from (4). Using (5), (6) for the process $G+Q \rightarrow Q^{\prime}+G^{\prime}$ we have with required accuracy:

$$
\begin{array}{r}
A_{G Q}^{Q^{\prime} G^{\prime}}=\frac{g^{2}}{-q^{2}} \bar{u}\left(p_{Q^{\prime}}\right) t^{G} t^{G^{\prime}}\left\{\hat{e}_{G} \hat{q} \hat{e}_{G^{\prime}}^{*}\left[1+\omega^{(1)}\left(q^{2}\right)\left(2 \delta_{e}^{(1)}+\frac{C_{F}}{N_{c}} \ln \frac{s}{-q^{2}}\right)\right]\right. \\
\left.+\omega^{(1)}\left(q^{2}\right) \delta_{q}^{(1)}\left[\hat{e}_{G}\left(e_{G^{\prime}}^{*} q\right)+\left(e_{G} q\right) \hat{e}_{G^{\prime}}^{*}\right]\right\} u\left(p_{Q}\right)
\end{array}
$$

where $q=\left(p_{Q}-p_{G^{\prime}}\right)$.

In the following in the amplitudes at the right hand side of (14) we denote Reggeized gluon and quark momenta $q_{1}$ and $q_{2}$ respectively, $q_{1}+q_{2}=q$, and Reggeized gluon colour index $r$. Since $q_{1} \simeq q_{1 \perp}, q_{2} \simeq q_{2 \perp}$, everywhere below we omit the sign $\perp$ at $q_{1,2}$, so that $q_{i}$ means $q_{i \perp}, \quad i=1,2$.

Amplitudes for processes $A B \rightarrow A^{\prime} B^{\prime}$ with gluon exchanges with our accuracy are written as

$$
\mathcal{A}_{A B}^{A^{\prime} B^{\prime}}=\frac{2 s}{q_{1}^{2}} \Gamma_{A^{\prime} A}^{r}\left(1+\omega^{(1)}\left(q_{1}^{2}\right) \ln \frac{s}{-q_{1}^{2}}\right) \Gamma_{B^{\prime} B}^{r},
$$

where $\Gamma_{A^{\prime} A}^{r}$ and $\Gamma_{B^{\prime} B}^{r}$ are Reggeized gluon vertices, which can be found in [12]. In the direct channel we need quark-gluon scattering amplitudes. For the process $G+Q \rightarrow G_{1}+Q_{1}$ (see 
the left part of Fig. 2a) we obtain

$$
\begin{aligned}
A_{G Q}^{G_{1} Q_{1}}=\frac{2 g^{2} s}{q_{1}^{2}} & \mathrm{~T}_{G_{1} G}^{r} \bar{u}\left(p_{Q_{1}}\right) t^{r} \frac{\hat{p}_{1}}{s} u\left(p_{Q}\right) \\
\times & \begin{array}{r}
\times-\left(e_{G_{1}}^{*} e_{G}\right)\left[1+\omega^{(1)}\left(q_{1}^{2}\right)\left(\delta_{G}^{(1+)}+\delta_{G}^{(1-)}+\delta_{Q}^{(1)}+\ln \frac{s}{-q_{1}^{2}}\right)\right] \\
\left.\quad+\omega^{(1)}\left(q_{1}^{2}\right)(D-2) \frac{\left(e_{G_{1}}^{*} q_{1}\right)\left(e_{G} q_{1}\right)}{q_{1}^{2}} \delta_{G}^{(1-)}\right\}
\end{array}
\end{aligned}
$$

where $\mathrm{T}_{G_{1} G}^{r}$ are colour generators in the adjoint representation, $q_{1}=\left(p_{G_{1}}-p_{G}\right)_{\perp}, \delta_{Q}$ represents the one loop corrections to the Quark-Quark-Reggeon vertex,

$$
\begin{aligned}
\delta_{Q}^{(1)}=\frac{1}{2}\left[\frac{1}{\epsilon}+\psi(1-\epsilon)+\psi(1)-2 \psi\right. & (1+\epsilon)+\frac{2+\epsilon}{2(1+2 \epsilon)(3+2 \epsilon)} \\
& \left.-\frac{1}{2 N_{c}^{2}}\left(1+\frac{2}{\epsilon(1+2 \epsilon)}\right)-\frac{n_{f}}{N_{c}} \frac{1+\epsilon}{(1+2 \epsilon)(3+2 \epsilon)}\right]
\end{aligned}
$$

$n_{f}$ is the number of quark flavours; $\delta_{G}^{(1+)}$ and $\delta_{G}^{(1+)}$ represent helicity conserving and helicity violating corrections to the Gluon-Gluon-Reggeon vertex;

$$
\begin{gathered}
\delta_{G}^{(1+)}=\frac{1}{2}\left[\frac{2}{\epsilon}+\psi(1-\epsilon)+\psi(1)-2 \psi(1+\epsilon)\right. \\
\left.-\frac{9(1+\epsilon)^{2}+2}{2(1+\epsilon)(1+2 \epsilon)(3+2 \epsilon)}+\frac{n_{f}}{N_{c}} \frac{(1+\epsilon)^{3}+\epsilon^{2}}{(1+\epsilon)^{2}(1+2 \epsilon)(3+2 \epsilon)}\right] \\
\delta_{G}^{(1-)}=\frac{\epsilon}{2(1+\epsilon)(1+2 \epsilon)(3+2 \epsilon)}\left(-1+\frac{n_{f}}{N_{c}(1+\epsilon)}\right) .
\end{gathered}
$$

Note, that in order to obtain $A_{Q_{1} G_{1}}^{Q^{\prime} G^{\prime}}$ (see the right part of Fig. 2b) from (18) one has to change $\hat{p}_{1}$ to $\hat{p}_{2}$ besides evident substitution of symbols .

In addition to presented amplitudes, in the crossed channel we need gluon-gluon and quark-antiquark forward scattering amplitudes (see Fig. 3).

The first (see the left part of Fig. 3a) is written as

$$
\begin{aligned}
A_{G \tilde{G}}^{G_{1} \tilde{G}_{1}}= & \frac{2 g^{2} s}{q_{1}^{2}} \mathrm{~T}_{G_{1} G}^{r} \mathrm{~T}_{\tilde{G}_{1} \tilde{G}}^{r}\left\{\left(e_{G_{1}}^{*} e_{G}\right)\left(e_{\tilde{G}_{1}}^{*} e_{\tilde{G}}\right)\left[1+\omega^{(1)}\left(q_{1}^{2}\right)\left(2 \delta_{G}^{(1+)}+2 \delta_{G}^{(1-)}+\ln \frac{s}{-q_{1}^{2}}\right)\right]\right. \\
& \left.-\omega^{(1)}\left(q_{1}^{2}\right)(D-2) \frac{\left(\left(e_{G_{1}}^{*} e_{G}\right)\left(e_{\tilde{G}_{1}}^{*} q_{1}\right)\left(e_{\tilde{G}} q_{1}\right)+\left(e_{G_{1}}^{*} q_{1}\right)\left(e_{G} q_{1}\right)\left(e_{\tilde{G}_{1}}^{*} e_{\tilde{G}}\right)\right)}{q_{1}^{2}} \delta_{G}^{(1-)}\right\}
\end{aligned}
$$

and the second (see the right part of Fig. 3b)

$$
A_{Q_{1} \bar{Q}_{1}}^{Q^{\prime} \bar{Q}^{\prime}}=-\frac{2 g^{2} s}{q_{1}^{2}} \bar{u}\left(p_{Q^{\prime}}\right) t^{r} \frac{\hat{p}_{2}}{s} u\left(p_{Q_{1}}\right) \bar{v}\left(p_{\bar{Q}_{1}}\right) t^{r} \frac{\hat{p}_{1}}{s} v\left(p_{\bar{Q}^{\prime}}\right)\left[1+\omega^{(1)}\left(q_{1}^{2}\right)\left(2 \delta_{Q}^{(1)}+\ln \frac{s}{-q_{1}^{2}}\right)\right] .
$$

Before to start with calculation let us show that only a colour triplet state survives in the discontinuity, due to cancellation of contributions of all other colour states in the direct and 
crossed channels. In fact, this cancellation has the same nature as in leading order [6]. The matter is that if a one-loop contribution is taken for one of the PPR vertices in Figs. 2 and 3 , then all other vertices must be taken at NLO in Born approximation. Therefore either both lower, or both upper vertices are Born ones. Let us consider the first case. Since upper parts of the diagrams Fig. 2a and Fig. 3a are equal, contributions to $M+M_{c}$ from the lower lines enter as the sum

$$
\gamma_{\perp}^{\mu} t^{G^{\prime}} \sum_{\lambda} u^{\lambda}\left(p_{Q_{1}}\right) \bar{u}^{\lambda}\left(p_{Q_{1}}\right) t^{r} \frac{\hat{p}_{1}}{s}-\sum_{\lambda} \hat{e}_{\tilde{G}_{1}}^{\lambda} t^{\tilde{G}_{1}}\left(e_{\tilde{G}_{1}}^{\lambda}\right)^{\star \mu} T_{\tilde{G}_{1} G^{\prime}}^{r}=\gamma_{\perp}^{\mu} t^{r} t^{G^{\prime}} .
$$

Here we have omitted terms with leftmost $\hat{p}_{1}$ because of the reason explained above, and have taken the same Lorentz and colour indices of the gluons $G^{\prime}$ and $\tilde{G}$ as we don't write their wave functions. It's easy to see that lower lines of Fig. $2 \mathrm{~b}$ and Fig. 3b give in sum the same result. Since $t^{r} t^{G^{\prime}}$ projects the $t$-channel quark-gluon state on a colour triplet, it means that contributions of another colour states cancel in the sum of the direct and cross channels. The case when both upper vertices are Born ones can be considered quite analogously. One can come to the same conclusion seeing that sum of contributions of Fig. 2a and Fig. 3b, as well as Fig. 2b and Fig. 3a, is proportional $t^{G} t^{r}$. It is not difficult to understand that the cancellation of colour states different from triplet is not restricted by considered diagrams and by the two-loop approximation, but is a general property of NLA, as well as LLA.

Since we have shown that only a colour triplet survives in the $t$-channel we can write

$$
\mathrm{P}_{3}^{(+)} \sum_{P_{1} P_{2}} \mathcal{A}_{G Q}^{P_{1} P_{2}}\left(\mathcal{A}_{Q^{\prime} G^{\prime}}^{P_{1} P_{2}}\right)^{*}=g^{4} \omega^{(1)}(t) s \bar{u}\left(p_{Q^{\prime}}\right) t^{G} t^{G^{\prime}} \mathcal{M}^{(2)} u\left(p_{Q}\right)
$$

With the amplitudes listed above calculation of $\mathcal{M}^{(2)}$ is straightforward. Dividing it into two pieces, $\mathcal{M}^{(2)}=\mathcal{M}_{Q}^{(2)}+\mathcal{M}_{G}^{(2)}$, one of which contains one-loop corrections for a quark channel, and another for a gluon channel, we obtain

$$
\mathcal{M}_{Q}^{(2)}=\left(\frac{q^{2}}{q_{2}^{2}}\right)^{-{ }^{-\epsilon} C_{F}} \frac{{ }^{2} q_{2}^{2}}{q_{1}^{2}}\left\{\left(2 \delta_{e}^{(1)}+\frac{C_{F}}{N_{c}} \ln \frac{s}{-q_{2}^{2}}\right) \hat{e}_{G} \hat{q}_{2} \hat{e}_{G^{\prime}}^{*}+\delta_{q}^{(1)}\left[\hat{e}_{G}\left(e_{G^{\prime}}^{*} q_{2}\right)+\left(e_{G} q_{2}\right) \hat{e}_{G^{\prime}}^{*}\right]\right\},
$$

and

$$
\begin{aligned}
\mathcal{M}_{G}^{(2)}=\left(\frac{q^{2}}{q_{1}^{2}}\right)^{-\epsilon} \frac{1}{q_{2}^{2} q_{1}^{2}}\left\{\left[-\frac{1}{N_{c}} \delta_{Q}^{(1)}+\right.\right. & \left.N_{c}\left(\delta_{G}^{(1+)}+\delta_{G}^{(1-)}\right)+C_{F} \ln \frac{s}{-q_{1}^{2}}\right] \hat{e}_{G} \hat{q}_{2} \hat{e}_{G^{\prime}}^{*} \\
& \left.-\frac{N_{c}}{2} \frac{D-2}{q_{1}^{2}} \delta_{G}^{(1-)}\left[\hat{e}_{G} \hat{q}_{2} \hat{q}_{1}\left(e_{G^{\prime}}^{*} q_{1}\right)+\left(e_{G} q_{1}\right) \hat{q}_{1} \hat{q}_{2} \hat{e}_{G^{\prime}}^{*}\right]\right\} .
\end{aligned}
$$

Integration over the phase space element $d \Phi_{2}=d^{D-2} q_{2} /\left(2 s(2 \pi)^{D-2}\right)$ is quite simple. The integrals are well convergent at large $\left|q_{2}\right|$, so that the integration region can be expanded to infinity. For convenience of a reader we present necessary formulas in Appendix A. As a result, we obtain for the two-particle contribution to $\Delta_{s}$

$$
\begin{aligned}
\Delta_{s}^{(2)}=\frac{2 N_{c} \epsilon\left(-q^{2}\right)^{1-\epsilon}}{\Gamma_{\epsilon} \pi^{(D-2) / 2}} \int d^{D-2} q_{2} \mathcal{M}^{(2)}=2 N_{c} \mathrm{X}_{\Gamma} & \left\{2 C_{F} \delta_{e}^{(1)}+\frac{N_{c}}{2}\left(\delta_{G}^{(1+)}-\frac{2 \epsilon}{1-\epsilon} \delta_{G}^{(1-)}\right)\right. \\
-\frac{1}{2 N_{c}} \delta_{Q}^{(1)}+\frac{C_{F}^{2}}{N_{c}}\left(\ln \frac{s}{-t}+\Psi_{1}\right)+ & \left.\frac{C_{F}}{2}\left(\ln \frac{s}{-t}+\Psi_{1}+\frac{1}{2 \epsilon}\right)\right\} \hat{\mathcal{Q}} \\
& +\mathrm{X}_{\Gamma}\left\{C_{F} \delta_{q}^{(1)}+N_{c} \frac{\epsilon(1+\epsilon)}{1-\epsilon} \delta_{G}^{(1-)}\right\} \hat{\mathcal{E}}
\end{aligned}
$$


where

$$
\mathrm{X}_{\Gamma}=\frac{\Gamma(1-2 \epsilon) \Gamma^{2}(1+2 \epsilon)}{\Gamma(1+\epsilon) \Gamma(1+3 \epsilon) \Gamma^{2}(1-\epsilon)},
$$

and

$$
\Psi_{1}=\psi(1-2 \epsilon)+\psi(1+3 \epsilon)-\psi(1+2 \epsilon)-\psi(1-\epsilon)
$$

\section{TREE-PARTICLE CONTRIBUTION TO THE DISCONTINUITY}

Let us denote intermediate particles in the unitarity condition (14) $P_{j}$ and their momenta $k_{j}, j=1 \div 3$. Just as before, only limited transverse momenta are important. As for longitudinal ones, let us put (without loss of generality) $\alpha_{3} \simeq 1$, that is for the direct process $G+Q \rightarrow Q^{\prime}+G^{\prime}$ a particle $P_{3}$ is produced in the fragmentation region of the initial quark (note that for the crossed process $G+\tilde{G} \rightarrow Q^{\prime}+\bar{Q}^{\prime}$ it is the region of $\tilde{G}$ fragmentation). Then $\beta_{1}+\beta_{2} \simeq 1$, i.e. at least one of particles $P_{i}, i=1,2$, is produced in the gluon fragmentation region. Let it will be $P_{1}$; then $\beta_{1} \sim 1$, but $\beta_{2}$ can vary from $\beta_{2} \sim 1$ (that means $P_{2}$ as well as $P_{1}$ is produced in the gluon fragmentation region) to $\beta_{2} \sim\left|k_{2 \perp}^{2}\right| / s$ (it means $\alpha_{2} \sim 1$, i.e. $P_{2}$ is in the quark fragmentation region). Of course, the same can be said with substitution $1 \leftrightarrow 2$. Note that region $1 \gg \beta_{i} \gg\left|k_{i \perp}^{2}\right| / s$ is usually called multi-Regge, or central region for a particle $P_{i}$. But this region does not require separate consideration, because amplitudes for production of $P_{i}$ in any of fragmentation regions are applicable in it. Actually, natural bounds for domains of applicability of these amplitudes are $\alpha_{i} \ll 1$ for the gluon and $\beta_{i} \ll 1$ for the quark fragmentation regions. Therefore, it is sufficient to consider

two regions: $1 \geq \beta_{i} \geq \sqrt{\left|k_{i \perp}^{2}\right| / s}$ and $1 \geq \alpha_{i} \geq \sqrt{\left|k_{i \perp}^{2}\right| / s}$. For a brevity, we will say that in the first (second) case there are two particles in the gluon (quark) fragmentation region. Moreover, the symmetry with respect to $\alpha \leftrightarrow \beta$ in the definition of the regions permits to consider only one of them. Indeed, as regards the inverse reaction $\left(Q^{\prime}+G^{\prime} \rightarrow G+Q\right)$ the names of the regions must be changed; therefore their contribution to the discontinuity are related by the substitutions $Q \leftrightarrow Q^{\prime}, G \leftrightarrow G^{\prime}$ and complex conjugation. We will consider the gluon fragmentation region.

In this region amplitudes in the right hand side of the unitarity relation (14) can be written, in accordance with our agreement about denotations, as

$$
\mathcal{A}_{A B}^{P_{1} P_{2} P_{3}}=\frac{2 s}{q_{1}^{2}} \Gamma_{\left\{P_{1} P_{2}\right\} A}^{r} \Gamma_{P_{3} B}^{r}
$$

and

$$
\mathcal{A}_{A B}^{P_{1} P_{2} P_{3}}=\Gamma_{\left\{P_{1} P_{2}\right\}} \frac{-\hat{q}_{2}}{q_{2}^{2}} \Gamma_{P_{3} B}
$$

for gluon and quark exchanges respectively, with the same vertices $\Gamma_{P_{3} B}^{r}$ and $\Gamma_{P_{3} B}$ as for the elastic amplitudes, but taken now in Born approximation only. Therefore, as well as in the two-particle contribution, only t-channel colour triplet does survive in positive signature, since

$$
\mathrm{P}^{(+)} \sum_{P_{3}} \Gamma_{P_{3} Q}^{r}\left(\Gamma_{P_{3} G^{\prime}}\right)^{*}=-\mathrm{P}^{(+)} \sum_{P_{3}} \Gamma_{P_{3} Q}\left(\Gamma_{P_{3} G^{\prime}}^{r}\right)^{*}=-\frac{g^{2}}{2} t^{r} t^{G^{\prime}} \hat{e}_{G^{\prime}}^{*} u\left(p_{Q}\right) .
$$

The vertices $\Gamma_{\left\{P_{1} P_{2}\right\} A}^{r}$ and $\Gamma_{\left\{P_{1} P_{2}\right\} A}$ can be found in Refs. [12] and [13] respectively. Actually, they can be easily calculated, since are given by Born amplitudes for processes $A+R \rightarrow$ $P_{1}+P_{2}$, where $R$ is either a gluon (for $\Gamma_{\left\{P_{1} P_{2}\right\} A}^{r}$ ) with momentum $q_{1}$, colour index $r$ and 
polarization vector $p_{2} / s\left(p_{1} / s\right)$ for a particle $A$ with predominant momentum components along $p_{1}\left(p_{2}\right)$, or a quark (for $\Gamma_{\left\{P_{1} P_{2}\right\} A}$ ) with momentum $q_{2}$ and omitted quark wave function. An important point is that corresponding light-cone gauge (see (3)) must be taken not only for real gluons, but for virtual ones as well. Note that the hermitian property of Born amplitudes gives the relations

$$
\left(\Gamma_{\left\{P_{1} P_{2}\right\} Q^{\prime}}^{r}\right)^{*}=\Gamma_{Q^{\prime}\left\{P_{1} P_{2}\right\}}^{r} \quad\left(\Gamma_{\left\{P_{1} P_{2}\right\} Q^{\prime}}\right)^{\dagger}=\Gamma_{Q^{\prime}\left\{P_{1} P_{2}\right\}} \gamma^{0}
$$

which we use in the following.

Let us denote

$$
\sum_{P_{1} P_{2}}\left(\Gamma_{\left\{P_{1} P_{2}\right\} G}^{r} \Gamma_{Q^{\prime}\left\{P_{1} P_{2}\right\}}-\Gamma_{\left\{P_{1} P_{2}\right\} G} \Gamma_{Q^{\prime}\left\{P_{1} P_{2}\right\}}^{r}\right) t^{r}=g^{4} \bar{u}\left(p_{Q^{\prime}}\right) t^{G} F_{G}
$$

The particles $P_{1}$ and $P_{2}$ can be two gluons $\left(G_{1} G_{2}\right)$, quark and gluon $\left(Q_{1} G_{2}\right)$, and quark and antiquark $\left(Q_{1} \bar{Q}_{2}\right)$. Evidently, only the first (second) term in the left hand side of (34) contributes in the first and third cases (in the second case). Using that the phase space element $d \Phi_{3}$ in the gluon fragmentation region looks like

$$
d \Phi_{3}=\delta\left(1-\beta_{1}-\beta_{2}\right) \frac{d \beta_{1} d \beta_{2}}{4 s \beta_{1} \beta_{2}} \frac{d^{D-2} k_{1} d^{D-2} k_{2}}{(2 \pi)^{2 D-3}},
$$

with the help of (30)-(32) we obtain the contribution of this region to $\Delta_{s}(14)$ in the form

$$
\begin{aligned}
\Delta_{s}^{(3) G}=-\frac{\epsilon^{2}\left(-q^{2}\right)^{1-2 \epsilon}}{\pi^{(D-2)} \Gamma_{\epsilon}^{2}} \int \frac{d \beta_{1} d \beta_{2}}{\beta_{1} \beta_{2}} \frac{d^{D-2} k_{1} d^{D-2} k_{2}}{q_{1}^{2} q_{2}^{2}} F_{G} \hat{q}_{2} \hat{e}_{G^{\prime}}^{*} \\
\quad \times \delta\left(1-\beta_{1}-\beta_{2}\right) \theta\left(\beta_{1}-\sqrt{\frac{\left|k_{1 \perp}^{2}\right|}{s}}\right) \theta\left(\beta_{2}-\sqrt{\frac{\left|k_{2 \perp}^{2}\right|}{s}}\right)
\end{aligned}
$$

where $q_{1}$ and $q_{2}$ are the momenta of $t$-channel gluon and quark respectively, $q_{1}+q_{2}=q$. As it was already pointed out, total three-particle contribution can be written then as

$$
\Delta_{s}^{(3)}=\Delta_{s}^{(3) G}+\bar{\Delta}_{s}^{(3) G}\left(G \rightarrow G^{\prime}\right)
$$

where $\bar{\Delta}=\gamma^{0} \Delta^{\dagger} \gamma^{0}$. Note that logarithms of $s$ appear in $\Delta_{s}^{(3) G}$ from integration over $\beta_{i}$ of those terms in $F_{G}$ which don't turn into zero at $\beta_{i} \rightarrow 0$. It is always possible to rewrite $F_{G}$ as a sum of terms which turn into zero either at $\beta_{1}=0$, or at $\beta_{2}=0$. For the first (second) of them the limitation $\beta_{1}>\sqrt{\left|k_{1 \perp}^{2}\right| / s}\left(\beta_{2}>\sqrt{\left|k_{2 \perp}^{2}\right| / s}\right)$ can be taken away; at that the change of variables $k_{1 \perp}=\beta_{1} l_{1 \perp}\left(k_{2 \perp}=\beta_{2} l_{2 \perp}\right)$ turn out often to be useful, and we meet integrals

$$
\int_{\sqrt{\left|k_{i \perp}^{2}\right| / s}}^{1} \frac{d \beta_{i}}{\beta_{i}}\left(1-\beta_{i}\right)^{\delta}=\frac{1}{2} \ln \frac{s}{\left|k_{i \perp}^{2}\right|}+\psi(1)-\psi(\delta+1),
$$

where $\delta$ is proportional to $\epsilon$.

Calculation of integrals without $\ln \left|k_{i \perp}^{2}\right|$ does not represent a problem. The list of basis integrals is presented in Appendix B. Contrary, two of integrals with $\ln \left|k_{i \perp}^{2}\right|$ can not be expressed in terms of elementary functions at arbitrary $\epsilon$. Besides of the integral $I_{0}$ (see 
(47)) encountered already in the calculations of the two-loop gluon Regge trajectory, here we meet a new nontrivial integral $I_{1}$ (see (48)) which is considered in Appendix C.

In the following we will use Eqs. (34), (36), (37) and the denotations:

$$
k_{i G}=\left(k_{i}-\beta_{i} p_{G}\right)_{\perp}, k_{i Q^{\prime}}=\left(k_{i}-\beta_{i} p_{Q^{\prime}}\right)_{\perp}, k_{12}=-k_{21}=\left(\beta_{2} k_{1}-\beta_{1} k_{2}\right)_{\perp} .
$$

It's easy to understand that $k_{i A}(i=1,2)$ is transverse part of $k_{i}$ with respect to $p_{A}, p_{2}$ plane, multiplied by $\beta_{A}$.

Since the integration in (36) is symmetric with respect to exchange $k_{1} \leftrightarrow k_{2}$, we will systematically omit in $F_{G}$ contributions antisymmetric relative to this exchange, without further reminding.

\subsection{Fragmentation into two gluons}

The vertex for production of the gluons $G_{1}, G_{2}$ with colour indices $i_{1}, i_{2}$ by the initial gluon $G$ can be written as

$$
\Gamma_{\left\{G_{1} G_{2}\right\} G}^{r}=g^{2}\left\{\left(T^{G} T^{r}\right)_{i_{1} i_{2}}\left[\gamma^{\mu \nu}\left(k_{1 G}\right)-\gamma^{\mu \nu}\left(k_{12}\right)\right] e_{1 \mu}^{*} e_{2 \nu}^{*}+(1 \leftrightarrow 2)\right\},
$$

where

$$
\gamma^{\mu \nu}(p)=\frac{2}{p^{2}}\left[\beta_{1} \beta_{2} g_{\perp}^{\mu \nu}\left(e_{G} p\right)-\beta_{1} e_{G}^{\mu} p^{\nu}-\beta_{2} p^{\mu} e_{G}^{\nu}\right] .
$$

The vertex for a quark exchange can be taken in [13]. Using Sudakov parametrization and omitting terms with rightmost $\hat{p}_{2}$ we have for it

$$
\Gamma_{Q^{\prime}\left\{G_{1} G_{2}\right\}}=-g^{2} \bar{u}\left(p_{Q^{\prime}}\right)\left\{t^{i_{1}} t^{i_{2}}\left[\gamma_{12}^{\mu \nu}-\gamma_{[12]}^{\mu \nu}\right] e_{1 \mu} e_{2 \nu}+(1 \leftrightarrow 2)\right\},
$$

where

$$
\begin{gathered}
\gamma_{12}^{\mu \nu}=\frac{1}{k_{1 Q^{\prime}}^{2}}\left(\beta_{1} \hat{k}_{1 Q^{\prime}} \gamma_{\perp}^{\mu}-2 k_{1 Q^{\prime}}^{\mu}\right) \gamma_{\perp}^{\nu}, \\
\gamma_{[12]}^{\mu \nu}=\frac{2}{k_{12}^{2}}\left[\beta_{1} \beta_{2} g_{\perp}^{\mu \nu} \hat{k}_{12}-\beta_{1} \gamma_{\perp}^{\mu} k_{12}^{\nu}-\beta_{2} k_{12}^{\mu} \gamma_{\perp}^{\nu}\right] .
\end{gathered}
$$

Note that lower indices of $\gamma^{\mu \nu}$-vertices in (41) are determined by sequences of colour matrices in corresponding group factors, and square brackets in $\gamma_{[12]}^{\mu \nu}$ emphasize its antisymmetry with respect to the permutation $1 \leftrightarrow 2$, as well as its relation with the colour factor $\left[t^{i_{1}} t^{i_{2}}\right]$.

As was pointed already, only the first term in the left side part of (34) does contribute in the case of two-gluon production. Putting there the vertices (40) and (41) and performing summation over spin and colour of intermediate gluons, after simple colour algebra we obtain for the two-gluon contribution to $F_{G}$

$$
F_{G}^{G G}=-\frac{N_{c}^{2}}{4}\left[\left(\gamma_{\mu \nu}\left(k_{1 G}\right)-\gamma_{\mu \nu}\left(k_{12}\right)\right)\left(\gamma_{12}^{\mu \nu}-\gamma_{[12]}^{\mu \nu}\right)+(1 \leftrightarrow 2)\right] .
$$

One should pay attention that all terms in (43) correspond to planar diagrams. It is an important property of a colour triplet state in the $t$-channel which strongly simplifies calculations. 
The convolutions entering in (43) give

$$
\begin{gathered}
\gamma_{\mu \nu}(p) \gamma_{12}^{\mu \nu}=\frac{2}{k_{1 Q^{\prime}}^{2} p^{2}}\left\{\hat{e}_{G}\left[2 \beta_{2}\left(k_{1 Q^{\prime}} p\right)+\beta_{1}\left(1-2 \beta_{2}\right) \hat{k}_{1 Q^{\prime}} \hat{p}\right]+4 \beta_{1} \beta_{2}\left(e_{G} k_{1 Q^{\prime}}\right) \hat{p}\right. \\
\left.+\beta_{1} \beta_{2}\left[\beta_{1}(D-2)-4\right]\left(e_{G} p\right) \hat{k}_{1 Q^{\prime}}\right\} ; \\
\gamma_{\mu \nu}(p) \gamma_{[12]}^{\mu \nu}=\frac{-4}{k_{12}^{2} p^{2}}\left\{\beta_{1} \beta_{2}\left(2-\beta_{1} \beta_{2}(D-2)\right)\left(e_{G} p\right) \hat{k}_{12}\right. \\
\left.-2 \beta_{1} \beta_{2}\left(e_{G} k_{12}\right) \hat{p}-\left(1-2 \beta_{1} \beta_{2}\right)\left(k_{12} p\right) \hat{e}_{G}\right\} .
\end{gathered}
$$

We obtain the two-gluon contribution in $\Delta_{s}^{(3) G}$ by substituting (43) in (36) and performing integration. Note that if we integrate over all phase space in (36), we have to take into account equivalence of produced gluons by the factor $1 / 2$ !. With account of the quark fragmentation region according to (37) we obtain, denoting the contributions related to the terms $\gamma_{\mu \nu}(p) \gamma_{N}^{\mu \nu}$ in $F_{G}^{Q Q}(43)$ as $\Delta_{N * p}$ :

$$
\begin{gathered}
\Delta_{12 * k_{1 G}}=\frac{N_{c}^{2}}{4}\left\{2\left[\frac{2}{\epsilon}-\frac{3}{1+2 \epsilon}+\Psi_{2}+\ln \frac{s}{-t}+\frac{5}{2 \epsilon} \mathrm{X}_{\Gamma}+\frac{1}{2}\left(I_{0}+I_{1}\right)\right] \hat{\mathcal{Q}}+\frac{2 \epsilon}{1+2 \epsilon} \hat{\mathcal{E}}\right\} ; \\
\Delta_{12 * k_{12}}=\frac{N_{c}^{2}}{4} \mathrm{X}_{\Gamma}\left\{2\left[\frac{3}{1+2 \epsilon}-\frac{3}{\epsilon}-\ln \frac{s}{-t}-\Psi\right] \hat{\mathcal{Q}}-\frac{2 \epsilon}{1+2 \epsilon} \hat{\mathcal{E}}\right\} ; \quad \Delta_{[12] * k_{12}}=0 ; \\
\Delta_{[12] * k_{1 G}}=-\frac{N_{c}^{2}}{4} \mathrm{X}_{\Gamma}\left\{2\left[\ln \frac{s}{-t}+\Psi+\frac{5}{2 \epsilon}-\frac{4}{1+2 \epsilon}+\frac{1}{(1-\epsilon)(1+2 \epsilon)(3+2 \epsilon)}\right] \hat{\mathcal{Q}}\right. \\
\left.-\frac{4 \epsilon^{2}}{(3+2 \epsilon)(1-\epsilon)(1+2 \epsilon)} \hat{\mathcal{E}}\right\}
\end{gathered}
$$

where

$$
\begin{aligned}
& I_{0}=-\frac{\epsilon^{2}\left(-q^{2}\right)^{1-2 \epsilon}}{\Gamma_{\epsilon}^{2} \pi^{(D-2)}} \int \frac{d^{D-2} k_{1} d^{D-2} k_{2} q^{2}}{k_{1 \perp}^{2}\left(k_{1}-q\right)_{\perp}^{2} k_{2 \perp}^{2}\left(k_{2}-q\right)_{\perp}^{2}} \ln \frac{q^{2}}{\left(k_{1}-k_{2}\right)_{\perp}^{2}}, \\
& I_{1}=-\frac{\epsilon^{2}\left(-q^{2}\right)^{1-2 \epsilon}}{\Gamma_{\epsilon}^{2} \pi^{(D-2)}} \int \frac{d^{D-2} k_{1} d^{D-2} k_{2}\left(k_{1}-k_{2}\right)_{\perp}^{2}}{k_{1 \perp}^{2}\left(k_{1}-q\right)_{\perp}^{2} k_{2 \perp}^{2}\left(k_{2}-q\right)_{\perp}^{2}} \ln \frac{q^{2}}{\left(k_{1}-k_{2}\right)_{\perp}^{2}} .
\end{aligned}
$$

Here and below we use denotations

$$
\Psi_{2}=2[\psi(1)-\psi(1+2 \epsilon)], \quad \Psi=\psi(1-2 \epsilon)+\psi(1+3 \epsilon)-\psi(1+\epsilon)-\psi(1)+\Psi_{2} .
$$

The contribution $\Delta_{s}^{G G}$ of fragmentation into two gluons to $\Delta_{s}^{(3)}$ is

$$
\Delta_{s}^{G G}=\Delta_{12 * k_{1 G}}+\Delta_{12 * k_{12}}+\Delta_{[12] * k_{1 G}} .
$$

\subsection{Fragmentation into quark and gluon}

Let us denote particles produced in the gluon fragmentation region $Q_{1}$ and $G_{2}$ and their momenta $k_{1}$ and $k_{2}$ respectively. The vertex $\Gamma_{\left\{Q_{1} G_{1}\right\} G}$ can be obtained by crossing and appropriate substitutions from $\Gamma_{Q^{\prime}\left\{G_{1} G_{2}\right\}}$ (41). We will represent it as

$$
\Gamma_{\left\{Q_{1} G_{2}\right\} G}=-g^{2} \bar{u}\left(k_{1}\right)\left\{t^{G} t^{i_{2}}\left[\gamma_{G 2}^{\nu}-\gamma_{[G 2]}^{\nu}\right] e_{2 \nu}^{*}+t^{i_{2}} t^{G}\left[\gamma_{2 G}^{\mu}+\gamma_{[G 2]}^{\mu}\right] e_{2 \mu}^{*}\right\},
$$


where $\gamma_{G 2}^{\nu}, \gamma_{[G 2]}^{\nu}$ and $\gamma_{2 G}^{\nu}$ are obtained from $\gamma_{12}^{\mu \nu}, \gamma_{[12]}^{\mu \nu}$ and $\gamma_{21}^{\nu \mu}$ respectively by substitutions

$$
\beta_{1} \rightarrow \frac{1}{\beta_{1}}, \quad \beta_{2} \rightarrow-\frac{\beta_{2}}{\beta_{1}}, \quad k_{1 Q^{\prime}} \rightarrow-\frac{k_{1 G}}{\beta_{1}}, k_{12} \rightarrow \frac{k_{2 G}}{\beta_{1}}, k_{2 Q^{\prime}} \rightarrow \frac{k_{12}}{\beta_{1}},
$$

and convolution with $e_{G}^{\mu}$. The vertex $\Gamma_{Q^{\prime}\left\{Q_{1} G_{2}\right\}}^{r}$ can be found in [12] and represented as

$$
\Gamma_{Q^{\prime}\left\{Q_{1} G_{2}\right\}}^{r}=g^{2} \bar{u}\left(p_{Q^{\prime}}\right) \frac{\hat{p}_{2}}{s}\left\{t^{i_{2}} t^{r}\left(L_{\mu}\left(k_{2 Q^{\prime}}\right)+L_{\mu}\left(k_{1 Q^{\prime}}\right)\right)+t^{r} t^{i_{2}}\left(L_{\mu}\left(k_{12}\right)-L_{\mu}\left(k_{1 Q^{\prime}}\right)\right)\right\} e_{2}^{\mu} u\left(k_{1}\right),
$$

where

$$
L^{\mu}(p)=\frac{\beta_{2} \hat{p} \gamma_{\perp}^{\mu}-2 p^{\mu}}{p^{2}} .
$$

In the case of quark-gluon production only the second term in the right hand side of (34) does contribute. Using vertices (51) and (53), we obtain for the quark-gluon contribution to $F_{G}$ after summation over spin and colour

$$
F_{G}^{Q G}=-\beta_{1} \sum_{i j} L_{\mu}\left(k_{i j}\right)\left[C_{G 2}^{i j} \gamma_{G 2}^{\mu}+C_{2 G}^{i j} \gamma_{2 G}^{\mu}+C_{[G 2]}^{i j} \gamma_{[G 2]}^{\mu}\right]
$$

where $i j$ takes on values $1 Q^{\prime}, 2 Q^{\prime}, 12$ and

$$
\begin{aligned}
& C_{G 2}^{1 Q^{\prime}}=\frac{1}{4}, C_{G 2}^{2 Q^{\prime}}=\frac{1}{4}\left(1+\frac{1}{N_{c}^{2}}\right), C_{G 2}^{12}=\frac{1}{4 N_{c}^{2}} ; \\
& C_{2 G}^{1 Q^{\prime}}=\frac{1}{4}, C_{2 G}^{2 Q^{\prime}}=\frac{1}{4 N_{c}^{2}}, \quad C_{2 G}^{12}=-\frac{1}{4}\left(1-\frac{1}{N_{c}^{2}}\right) ; \\
& C_{[G 2]}^{1 Q^{\prime}}=0, C_{[G 2]}^{2 Q^{\prime}}=-\frac{1}{4}, \quad C_{[G 2]}^{12}=-\frac{1}{4} .
\end{aligned}
$$

Note that the term $C_{G 2}^{2 Q^{\prime}} L_{\mu}\left(k_{2 Q^{\prime}}\right) \gamma_{G 2}^{\mu}$ here corresponds to a non-planar diagram and leads to a complicated integral. Fortunately, it is cancelled by respective contribution from quarkantiquark production, as we will see in the next subsection.

For the products $L_{\mu}(p) \gamma_{m n}^{\mu}$ we have after some algebra:

$$
\begin{aligned}
L_{\mu}(p) \gamma_{G 2}^{\mu} & =\frac{1}{k_{1 G}^{2} p^{2}}\left\{2 \beta_{1} \beta_{2}(D-2)\left(e_{G} k_{1 G}\right) \hat{p}-\beta_{2}(D-6) \hat{p} \hat{k}_{1 G} \hat{e}_{G}-2 \hat{e}_{G} \hat{k}_{1 G} \hat{p}\right\} ; \\
L_{\mu}(p) \gamma_{2 G}^{\mu} & =\frac{1}{k_{12}^{2} p^{2}}\left\{\beta_{2}\left(\beta_{2}(D-2)-4\right) \hat{p} \hat{k}_{12}+4\left(k_{12} p\right)\right\} \hat{e}_{G} ; \\
L_{\mu}(p) \gamma_{[G 2]}^{\mu} & =\frac{2 \beta_{2}}{k_{2 G}^{2} p^{2}}\left\{\left(\frac{\beta_{2}}{\beta_{1}}-1\right) \hat{e}_{G} \hat{p} \hat{k}_{2 G}+\left(\beta_{2}(D-2)-4\right)\left(e_{G} k_{2 G}\right) \hat{p}\right. \\
& \left.+\frac{2}{\beta_{2}}\left(k_{2 G} p\right) \hat{e}_{G}+4\left(e_{G} p\right) \hat{k}_{2 G}\right\} .
\end{aligned}
$$

The quark-gluon contribution to $\Delta_{s}^{(3) G}$ is given by (36) with (55) instead of $F_{G}$. Denoting $\Delta_{m n \cdot i j}$ the contributions proportional $C_{m n}^{i j}$ in (55), after integration we obtain for them with account of the quark fragmentation region according to (37)

$$
\begin{aligned}
& \Delta_{G 2 \cdot 1 Q^{\prime}}=\frac{1}{4}\left\{2\left[\frac{3-2 \epsilon}{1+2 \epsilon}-\Psi_{2}-\ln \frac{s}{-t}-\frac{5}{2 \epsilon} \mathrm{X}_{\Gamma}-\frac{1}{2}\left(I_{0}+I_{1}\right)\right] \hat{\mathcal{Q}}+\frac{2 \epsilon}{1+2 \epsilon} \hat{\mathcal{E}}\right\} ; \\
& \Delta_{G 2 \cdot 12}=\frac{1}{4 N_{c}^{2}} \mathrm{X}_{\Gamma}\left\{-2\left[\ln \frac{s}{-t}+\Psi+\frac{1}{\epsilon}-\frac{3-2 \epsilon}{1+2 \epsilon}\right] \hat{\mathcal{Q}}+\frac{2 \epsilon}{1+2 \epsilon} \hat{\mathcal{E}}\right\}
\end{aligned}
$$




$$
\begin{gathered}
\Delta_{2 G \cdot 1 Q^{\prime}}=\frac{1}{4} \mathrm{X}_{\Gamma}\left\{2\left[\ln \frac{s}{-t}+\Psi+\frac{3}{2 \epsilon}-\frac{3}{2(1+2 \epsilon)}\right]\right\} \hat{\mathcal{Q}} ; \\
\Delta_{2 G \cdot 2 Q^{\prime}}=\frac{1}{4 N_{c}^{2}} \mathrm{X}_{\Gamma}\left\{2\left[\frac{3-2 \epsilon}{2(1+2 \epsilon)}-\frac{1}{\epsilon}\right]\right\} \hat{\mathcal{Q}} ; \quad \Delta_{2 G \cdot 12}=0 ; \\
\Delta_{[G 2] \cdot 2 Q^{\prime}}=\frac{1}{4}\left\{-2\left[-\frac{3}{1+2 \epsilon}+\frac{2}{\epsilon}+\Psi_{2}+\ln \frac{s}{-t}-\frac{\mathrm{X}_{\Gamma}}{\epsilon}-\frac{1}{2} I_{1}\right] \hat{\mathcal{Q}}-\frac{2 \epsilon}{1+2 \epsilon} \hat{\mathcal{E}}\right\} ; \\
\Delta_{[G 2] \cdot 12}=\frac{1}{4} \mathrm{X}_{\Gamma}\left\{2\left[\ln \frac{s}{-t}+\Psi+\frac{3}{\epsilon}-\frac{3}{1+2 \epsilon}\right] \hat{\mathcal{Q}}+\frac{2 \epsilon}{1+2 \epsilon} \hat{\mathcal{E}}\right\} .
\end{gathered}
$$

The contribution $\Delta_{G 2 \cdot 2 Q^{\prime}}$ is not presented here, since it is cancelled with respective contribution from quark-antiquark production, as was pointed out. The quark-gluon contribution to $\Delta_{s}^{(3)}$ is given by

$$
\Delta_{s}^{Q G}=\sum_{i j, m n} \Delta_{m n \cdot i j}, \quad i j=1 Q^{\prime}, 2 Q^{\prime}, 12 ; \quad m n=G 2,2 G,[G 2] .
$$

\subsection{Fragmentation into quark-antiquark pair}

As well as in the two-gluon case, only the first term in (34) exists. We denote momenta of particles $Q_{1}$ and $\bar{Q}_{2}$ in the gluon fragmentation region $k_{1}$ and $k_{2}$ respectively. The vertex $\Gamma_{\left\{Q_{1} \bar{Q}_{2}\right\} G}^{r}$ can be obtained by crossing and corresponding substitutions from $\Gamma_{Q^{\prime}\left\{Q_{1} G_{2}\right\}}^{r}(53)$ :

$$
\Gamma_{\left\{Q_{1} \bar{Q}_{2}\right\} G}^{r}=g^{2} \bar{u}\left(k_{1}\right) \frac{\hat{p}_{2}}{s}\left\{t^{G} t^{r}\left(L\left(k_{1 G}\right)-L\left(k_{12}\right)\right)+t^{r} t^{G}\left(L\left(k_{12}\right)+L\left(k_{2 G}\right)\right)\right\} v\left(k_{2}\right),
$$

where

$$
L(p)=\frac{-\hat{p} \hat{e}_{G}+2 \beta_{1}\left(p e_{G}\right)}{p^{2}} .
$$

The vertex $\Gamma_{Q^{\prime}\left\{Q_{1} \bar{Q}_{2}\right\}}$ can be obtained from [13]; a direct calculation does not encounter difficulties as well. We have

$$
\Gamma_{Q^{\prime}\left\{Q_{1} \bar{Q}_{2}\right\}}=g^{2}\left[\bar{v}\left(k_{2}\right) t^{c} \gamma^{\sigma} u\left(k_{1}\right) \bar{u}\left(p_{Q^{\prime}}\right) t^{c} A_{\sigma}-\bar{u}\left(p_{Q^{\prime}}\right) t^{c} \gamma^{\sigma} u\left(k_{1}\right) \cdot \bar{v}\left(k_{2}\right) t^{c} B_{\sigma} \delta_{Q_{1} Q}\right],
$$

where $\delta_{Q_{1} Q}$ shows that the last contribution exists only when an intermediate quark has the same flavour as the initial one, and the values $A_{\sigma}$ and $B_{\sigma}$ can be written as

$$
A^{\sigma}=\frac{-\beta_{1} \beta_{2}}{k_{12}^{2}}\left(\gamma_{\perp}^{\sigma}-\left(\hat{k}_{1}+\hat{k}_{2}\right)_{\perp} \frac{2 p_{2}^{\sigma}}{s}\right) ; B^{\sigma}=\frac{\beta_{1}}{k_{1 Q^{\prime}}^{2}}\left(\gamma_{\perp}^{\sigma}+\left(\hat{k}_{1}-\hat{p}_{Q^{\prime}}\right)_{\perp} \frac{2 p_{2}^{\sigma}}{s \beta_{2}}\right)
$$

By substituting the vertices (60) and (62) into (34), after summation over spin and colour states of intermediate particles we obtain for the quark-antiquark contribution to $F_{G}$

$$
F_{G}^{Q Q}=\sum_{i j}\left[C_{A}^{i j} \operatorname{tr}\left(\hat{k}_{1} \frac{\hat{p}_{2}}{s} L\left(k_{i j}\right) \hat{k}_{2} \gamma_{\sigma}\right) A^{\sigma}-C_{B}^{i j} \gamma_{\sigma} \hat{k}_{1} \frac{\hat{p}_{2}}{s} L\left(k_{i j}\right) \hat{k}_{2} B^{\sigma}\right]
$$


where $i j$ takes on values $1 G, 2 G, 12$ and

$$
\begin{array}{lll}
C_{A}^{1 G}=\frac{N_{c}}{8} n_{f}, & C_{A}^{2 G}=-\frac{N_{c}}{8} n_{f}, & C_{A}^{12}=-\frac{N_{c}}{4} n_{f} ; \\
C_{B}^{1 G}=\frac{1}{4 N_{c}^{2}}, & C_{B}^{2 G}=\frac{1}{4}\left(1+\frac{1}{N_{c}^{2}}\right), & C_{B}^{12}=\frac{1}{4} .
\end{array}
$$

Taking into account, as usually, external Dirac spinors, we obtain

$$
\begin{gathered}
\operatorname{tr}\left(\hat{k}_{1} \frac{\hat{p}_{2}}{s} L(p) \hat{k}_{2} \gamma_{\sigma}\right) A^{\sigma}=\frac{2 \beta_{1} \beta_{2}}{p^{2} k_{12}^{2}}\left(\left(k_{12} p\right) \hat{e}_{G}-\left(e_{G} k_{12}\right) \hat{p}+\left(1-4 \beta_{1} \beta_{2}\right)\left(e_{G} p\right) \hat{k}_{12}\right) \\
\gamma_{\sigma} \hat{k}_{1} \frac{\hat{p}_{2}}{s} L(p) \hat{k}_{2} B^{\sigma}=\frac{-\beta_{1}}{k_{1 Q^{\prime}}^{2} p^{2}}\left\{2 \beta_{1} \beta_{2}(D-2)\left(e_{G} p\right) \hat{k}_{1 Q^{\prime}}-\beta_{2}(D-6) \hat{k}_{1 Q^{\prime}} \hat{e}_{G}-2 \hat{e}_{G} \hat{p} \hat{k}_{1 Q^{\prime}}\right\} .
\end{gathered}
$$

The quark-antiquark contribution to $\Delta_{s}^{(3) G}$ is given by (36) with (64) instead of $F_{G}$. As have been mentioned above, in $F_{G}^{Q Q}$ we also have a term corresponding to a non-planar diagram. In (64) it stands with the coefficient $C_{B}^{2 G}$. Note, that $C_{B}^{2 G}=C_{G 2}^{2 Q^{\prime}}$ (see (56)). Moreover, comparing the first equations in (57) and the second in (66), one can see that the substitution

$$
k_{1 Q^{\prime}} \rightarrow-k_{2 Q^{\prime}}, \quad k_{2 G} \rightarrow-k_{1 G}
$$

turns $\gamma_{\sigma} \hat{k}_{1}\left(\hat{p}_{2} / s\right) L\left(k_{2 G}\right) \hat{k}_{2} B^{\sigma}$ into $\beta_{1} L_{\mu}\left(k_{2 Q^{\prime}}\right) \gamma_{G 2}^{\mu}$ with opposite sign. Note that for the quarkantiquark contribution $q_{2}$ in (36) is equal $p_{Q^{\prime} \perp}-k_{1 \perp}-k_{2 \perp}=\beta_{2} q-k_{1 Q^{\prime}}-k_{2 G}$. At the substitution (67) it turns into $\beta_{2} q+k_{2 Q^{\prime}}+k_{1 G}=k_{1 \perp}+k_{2 \perp}-p_{G \perp}$, that is just the $t$ channel quark momentum for the quark-gluon contribution. An important point is that the theta-functions in (36) can be omitted for the contributions of the nonplanar diagrams due to convergence of integrals, after that the substitution (67) does not influence on the integration region. Therefore these contributions cancel each other.

Performing integration and denoting $\Delta_{A \cdot i j}$ and $\Delta_{B \cdot i j}$ the contributions to (64) proportional $C_{A}^{1 j}$ and $C_{B}^{1 j}$ respectively, we obtain with account of the quark fragmentation region according to (37):

$$
\begin{gathered}
\Delta_{A \cdot 1 G}=\frac{N_{c}}{8} n_{f} \mathrm{X}_{\Gamma}\left\{\frac{-2}{1+2 \epsilon}\left(1-\frac{1}{\left(1-\epsilon^{2}\right)(3+2 \epsilon)}\right) \hat{\mathcal{Q}}-\frac{4 \epsilon^{2}}{\left(1-\epsilon^{2}\right)(1+2 \epsilon)(3+2 \epsilon)} \hat{\mathcal{E}}\right\} ; \\
\Delta_{B \cdot 1 G}=\frac{1}{4 N_{c}^{2}}\left\{2\left[-\frac{3-2 \epsilon}{1+2 \epsilon}+\Psi_{2}+\ln \frac{s}{-t}-\frac{\mathrm{X}_{\Gamma}}{\epsilon}-\frac{1}{2} I_{1}\right] \hat{\mathcal{Q}}-\frac{2 \epsilon}{1+2 \epsilon} \hat{\mathcal{E}}\right\} ; \\
\Delta_{B \cdot 12}=\frac{1}{4} \mathrm{X}_{\Gamma}\left\{-2\left[\frac{3-2 \epsilon}{1+2 \epsilon}-\ln \frac{s}{-t}-\Psi-\frac{1}{\epsilon}\right] \hat{\mathcal{Q}}-\frac{2 \epsilon}{1+2 \epsilon} \hat{\mathcal{E}}\right\} .
\end{gathered}
$$

Therefore, the quark-antiquark contribution to $\Delta_{s}^{(3)}$ can be written as

$$
\Delta_{s}^{Q Q}=2 \Delta_{A \cdot 1 G}+\Delta_{B \cdot 1 G}+\Delta_{B \cdot 12}+\Delta_{B \cdot 2 G},
$$

where $\Delta_{B \cdot 2 G}=-\Delta_{G 2 \cdot 2 Q^{\prime}}$, as it was shown above. Because of cancellation of these contributions in

$$
\Delta_{s}^{(3)}=\Delta_{s}^{G G}+\Delta_{s}^{G Q}+\Delta_{s}^{Q Q}
$$

$\Delta_{B \cdot 2 G}$ is not presented in (68), as well as $\Delta_{G 2 \cdot 2 Q^{\prime}}$ in (58). 


\section{TWO-LOOP CORRECTION TO THE QUARK TRAJECTORY}

The total discontinuity $\Delta_{s}$ is given by the sum of the contributions of two- and threeparticle intermediate states in the unitarity relation. The two-particle contribution $\Delta_{s}^{(2)}$ is given explicitly by (27). All necessary contributions to $\Delta_{s}^{(3)}(70)$ are calculated in the preceding section. They are given by (50)and (46) for fragmentation into two gluons, by (59) and (58) for fragmentation into quark and gluon, and by (69), (68) for fragmentation into quark and antiquark. Now we can compare the calculated discontinuity with the form (11) required by the quark Reggezation. First of all we note coincidence of the terms with $\ln s$. Actually this coincidence must be expected, since it is required by the quark Reggeization in LLA, which was already checked on this level. Much more important is that the calculated discontinuity has the same helicity structure as $\Delta_{R}(11)$, with the same coefficient at the structure $\hat{\mathcal{E}}$. It is a serious argument in favour of validity of the Reggeization hypothesis in NLA. Then comparing coefficients at the structure $\hat{\mathcal{Q}}$ we obtain

$$
\begin{aligned}
\delta_{\mathrm{T}}^{(2)}=C_{F}\left\{-n_{f}\right. & \frac{\mathrm{X}_{\Gamma}(1+\epsilon)}{(1+2 \epsilon)(3+2 \epsilon)}+N_{c}\left(\frac{1}{2} I_{0}+I_{1}-\mathrm{X}_{\Gamma}[\psi(1+\epsilon)-\psi(1+2 \epsilon)]\right. \\
+ & \left.\frac{7 \mathrm{X}_{\Gamma}}{2 \epsilon}+\frac{\mathrm{X}_{\Gamma}(11+7 \epsilon)}{2(1+2 \epsilon)(3+2 \epsilon)}\right)+2 C_{F}\left(-\frac{1}{2} I_{1}+\psi(1)-\psi(1-\epsilon)\right. \\
& \left.\left.+\left(2-\mathrm{X}_{\Gamma}\right)[\psi(1+\epsilon)-\psi(1+2 \epsilon)]-\frac{1+\mathrm{X}_{\Gamma}}{\epsilon}-\frac{\left(1-\mathrm{X}_{\Gamma}\right)(3-\epsilon)}{2(1+2 \epsilon)}\right)\right\}
\end{aligned}
$$

where $\mathrm{X}_{\Gamma}$ is given by (28), $I_{0}$ and $I_{1}$ are defined in (47) and (48).

The two-loop corrections to the quark Regge trajectory $j_{Q}=1 / 2+\delta_{\mathrm{T}}(t)$ is determined by Eqs. (9), (71) at arbitrary space-time dimension $D=4+2 \epsilon$. Unfortunately, at arbitrary $D$ the integrals $I_{0}$ and $I_{1}$ can not be written in terms of elementary functions. In the limit $\epsilon \rightarrow 0$ we have for them (see Appendix $\mathbf{C}$ )

$$
I_{0}=\frac{1}{\epsilon}+15 \psi^{(2)}(1) \epsilon^{2}+\mathcal{O}\left(\epsilon^{3}\right), \quad I_{1}=-\frac{4}{\epsilon}+6 \psi^{(2)}(1) \epsilon^{2}+\mathcal{O}\left(\epsilon^{3}\right),
$$

where $\psi^{(2)}$ means the second derivative of the $\psi$-function. With this result and with the proportion $\psi^{(2)}(1)=-2 \zeta_{3}$, where $\zeta_{n}$ is the Riemann Zeta-function, from Eq.(71) we obtain for the two-loop correction up to non-vanishing at $\epsilon \rightarrow 0$ terms

$$
\delta_{\mathrm{T}}^{(2)}=C_{F}\left\{\beta_{0}-K \epsilon+\left(\left(\frac{202}{27}-9 \zeta_{3}\right) N_{c}-\frac{28}{27} n_{f}+8 \zeta_{3} C_{F}\right) \epsilon^{2}\right\}+\mathcal{O}\left(\epsilon^{3}\right),
$$

where

$$
\beta_{0}=\frac{11}{6} N_{c}-\frac{2}{3} n_{f}, \quad K=\left(\frac{67}{18}-\zeta_{2}\right) N_{c}-\frac{5}{9} n_{f},
$$

in agreement with the result of Ref. [16].

\section{SUMMARY}

In this paper we have checked compatibility of the quark Reggeization hypothesis with the $s$-channel unitarity by the explicit two-loop calculations and have found in the case 
of massless quarks the two-loop correction to the quark trajectory at arbitrary space-time dimension $D=4+2 \epsilon$. The $\epsilon$-expansion of the correction gives the result obtained in [16]. We have calculated the two-loop $s$-channel discontinuity of the backward quark-gluon scattering amplitude with positive signature keeping non-logarithmic terms and have proved that only a colour triplet part of the amplitude does survive at NLO as well as at LO. It was shown that the calculated discontinuity has a form required by the Reggeization hypothesis. The two-loop correction to the trajectory has been obtained from comparison of the calculated discontinuity with the Reggeized form. In the case of massive quarks the trajectory can be found by the same method, since all necessary quantities for such calculation are known.

The cancellation of contributions of colour states different from triplet in positive signature is not restricted by the two-loop approximation, but is a general property of NLA. Therefore in this approximation, as well as in LLA, real parts of amplitudes with positive signature are completely determined by Reggeized quark contributions.

Note that testing of the quark Reggeization performed up to now is rather limited. Even in LLA self-consistency of the hypothesis was shown only in a particular case of elastic quark-gluon scattering, although in all orders of $\alpha_{s}$. In NLA it is tested in the same process only in order $\alpha_{s}^{2}$. A possible way of more strict testing and, in principle, a complete proof is examination of "bootstrap" conditions on the Reggeized quark vertices and the trajectory in QCD. These conditions appear from comparison of Reggeized form of discontinuities of amplitudes with quark exchanges with the discontinuities calculated with use of the $s$-channel unitarity.

We have used dimensional regularization for both infrared and ultraviolet divergences and the bare coupling constant $g=g_{s} \mu^{\epsilon}$, so that besides of infrared poles in $\epsilon$ our result contains the ultraviolet poles. To remove them it is sufficient to express the bare coupling through renormalized one. In the $\overline{\mathrm{MS}}$ renormalization scheme

$$
g=g_{\mu} \mu^{-\epsilon}\left\{1+\beta_{0} \frac{g_{\mu}^{2} \Gamma(1-\epsilon)}{\epsilon(4 \pi)^{2+\epsilon}}\right\},
$$

where $g_{\mu}$ is the renormalized coupling at the normalization point $\mu$.

\section{ACKNOWLEDGMENTS}

A.V.B. grateful to M.I. Kotsky for helpful discussions. V.S.F. thanks the Alexander von Humboldt foundation for the research award, the Universität Hamburg and DESY, the Dipartimento di Fisica dell'Università della Calabria and the Istituto Nazionale di Fisica Nucleare - gruppo collegato di Cosenza for their warm hospitality while a part of this work was done. This work is supported in part by INTAS and in part by the Russian Fund of Basic Researches.

\section{APPENDIX A}

For convenience of a reader we list here the integrals encountered at the calculation of $\Delta_{s}^{(2)}$. Note that everywhere below we use Euclidean transverse momenta and omit the 
transversality sign.

$$
\begin{gathered}
J_{1}^{i}=\int \frac{d^{D-2} k k^{i}}{\left(k^{2}\right)^{1-\epsilon}(k-q)^{2}}=q^{i} \frac{\pi^{(D-2) / 2}}{\epsilon\left(q^{2}\right)^{1-2 \epsilon}} \Gamma_{\epsilon} \cdot \mathrm{X}_{\Gamma} \\
J_{2}^{i}=\int \frac{d^{D-2} k k^{i}}{\left(k^{2}\right)^{1-\epsilon}(k-q)^{2}} \ln \frac{s}{k^{2}}=J_{1}^{i}\left[\ln \frac{s}{-t}+\Psi_{1}\right] \\
J_{3}^{i}=\int \frac{d^{D-2} k q^{i}}{\left(k^{2}\right)^{1-\epsilon}(k-q)^{2}}=\frac{3}{2} J_{1}^{i} ; \\
J_{4}^{i}=\int \frac{d^{D-2} k q^{i}}{\left(k^{2}\right)^{1-\epsilon}(k-q)^{2}} \ln \frac{s}{k^{2}}=\frac{3}{2} J_{2}^{i}+\frac{1}{4 \epsilon} J_{1}^{i} ; \\
J_{5}^{\mu}=\int \frac{d^{D-2} k k^{l} k^{m} q^{i}}{\left(k^{2}\right)^{2-\epsilon}(k-q)^{2}}=\frac{1}{\epsilon(1-\epsilon)}\left(\frac{q^{k} q^{l}}{q^{2}}(1-2 \epsilon)+\frac{\delta^{k l}}{4}\right) J_{1}^{i} .
\end{gathered}
$$

Remind that

$$
\begin{gathered}
\Gamma_{\epsilon}=\frac{\Gamma^{2}(1+\epsilon) \Gamma(1-\epsilon)}{\Gamma(1+2 \epsilon)} ; \quad \mathrm{X}_{\Gamma}=\frac{\Gamma(1-2 \epsilon) \Gamma^{2}(1+2 \epsilon)}{\Gamma(1+\epsilon) \Gamma(1+3 \epsilon) \Gamma^{2}(1-\epsilon)} ; \\
\Psi_{1}=\psi(1+3 \epsilon)+\psi(1-2 \epsilon)-\psi(1+2 \epsilon)-\psi(1-\epsilon) .
\end{gathered}
$$

\section{APPENDIX B}

Apart from $I_{0}(47)$ and $I_{1}(48)$, considered in the next Appendix, integrals required for calculation of $\Delta_{s}^{(3)}$ can be transformed to ones listed below. Using the denotations (A6), (49) and

$$
\Lambda=\frac{\pi^{(D-2)}}{\epsilon^{2}\left(q^{2}\right)^{1-2 \epsilon}}
$$

we have

$$
\begin{gathered}
K_{1}=\int \frac{d k^{(D-2)} d p^{(D-2)}}{\Lambda\left(\Gamma_{\epsilon}\right)^{2}} \frac{\hat{e}_{G}(k q) \hat{p}}{k^{2}(k-q)^{2} p^{2}(p-q)^{2}}=\hat{e}_{G} \hat{q} \\
K_{2}=\int \frac{d k^{(D-2)} d p^{(D-2)}}{\Lambda\left(\Gamma_{\epsilon}\right)^{2}} \frac{\left(e_{G} k\right)(\hat{k}-\hat{q}) \hat{p}}{k^{2}(k-q)^{2} p^{2}(p-q)^{2}} \\
=-\hat{e}_{G} \hat{q} \frac{1}{2(1+2 \epsilon)}+\left(e_{G} q\right) \frac{\epsilon}{1+2 \epsilon} ; \\
K_{3}=\int \frac{d k^{(D-2)} d p^{(D-2)} \frac{\hat{e}_{G} \hat{k}(\hat{k}-\hat{q}) \hat{p}}{k^{2}(k-q)^{2} p^{2}(p-q)^{2}} \ln \frac{s}{(k-p)^{2}}}{=\hat{e}_{G} \hat{q}\left[\frac{1}{2}\left(I_{0}+I_{1}\right)+\mathrm{X}_{\Gamma} \frac{5}{2 \epsilon}+\ln \frac{s}{-t}\right] ;} \\
K_{4}=\int \frac{d k^{(D-2)} d p^{(D-2)}}{\Lambda\left(\Gamma_{\epsilon}\right)^{2}} \frac{\hat{e}_{G}(\hat{k}-\hat{q}) \hat{k} \hat{p}}{k^{2}(k-q)^{2} p^{2}(p-q)^{2}} \ln \frac{s}{(k-p)^{2}}=\hat{e}_{G} \hat{q}\left[-\frac{1}{2} I_{1}-\frac{\mathrm{X}_{\Gamma}}{\epsilon}+\ln \frac{s}{-t}\right]
\end{gathered}
$$




$$
\begin{gathered}
K_{5}=\int \frac{d k^{(D-2)} d p^{(D-2)}}{\Lambda\left(\Gamma_{\epsilon}\right)^{2}} \frac{\left(e_{G} k\right) \hat{k} \hat{p}}{k^{2}(k-p)^{2} p^{2}(p-q)^{2}}=-\left(\hat{e}_{G} \hat{q} \frac{1}{2}+\left(e_{G} q\right)(1+\epsilon)\right) \frac{\mathrm{X}_{\Gamma}}{(1+2 \epsilon)} \\
K_{6}=\int \frac{d k^{(D-2)} d p^{(D-2)}}{\Lambda\left(\Gamma_{\epsilon}\right)^{2}} \frac{\left(e_{G} k\right)(\hat{k}-\hat{p})(\hat{p}-\hat{q})}{k^{2}(k-p)^{2} p^{2}(p-q)^{2}}=\left(\hat{e} \hat{e}_{G} \hat{q} \frac{1}{4}+\left(e_{G} q\right) \epsilon^{2}\right) \frac{\mathrm{X}_{\Gamma}}{(1-\epsilon)(1+2 \epsilon)} \\
K_{7}=\int \frac{d k^{(D-2)} d p^{(D-2)}}{\Lambda\left(\Gamma_{\epsilon}\right)^{2}} \frac{\hat{e}_{G} \hat{k} p^{2}}{k^{2}(k-p)^{2} p^{2}(p-q)^{2}}=\hat{e}_{G} \hat{q} \mathrm{X}_{\Gamma} ; \\
K_{8}=\int \frac{d k^{(D-2)} d p^{(D-2)}}{\Lambda\left(\Gamma_{\epsilon}\right)^{2}} \frac{\hat{e}_{G} \hat{k} p^{2}}{k^{2}(k-p)^{2} p^{2}(p-q)^{2}} \ln \frac{s}{(k-p)^{2}} \\
=\hat{e}_{G} \hat{q}\left[\ln \frac{s}{-t}+\Psi-\Psi_{2}+\frac{1}{\epsilon}\right] \mathrm{X}_{\Gamma} ; \\
K_{9}=\int \frac{d k^{(D-2)} d p^{(D-2)}}{\Lambda\left(\Gamma_{\epsilon}\right)^{2}} \frac{\hat{e}_{G}(k p)(\hat{p}-\hat{q})}{k^{2}(k-p)^{2} p^{2}(p-q)^{2}}=-\frac{1}{2} \hat{e}_{G} \hat{q} \mathrm{X}_{\Gamma} \\
K_{10}=\int \frac{d k^{(D-2)} d p^{(D-2)}}{\Lambda\left(\Gamma_{\epsilon}\right)^{2}} \frac{\hat{e}_{G}(k p)(\hat{p}-\hat{q})}{k^{2}(k-p)^{2} p^{2}(p-q)^{2}} \ln \frac{s}{(k-p)^{2}} \\
=-\frac{1}{2} \hat{e}_{G} \hat{q}\left[\ln \frac{s}{-t}+\Psi-\Psi_{2}+\frac{3}{2 \epsilon}\right] \mathrm{X}_{\Gamma} \cdot
\end{gathered}
$$

\section{APPENDIX C}

At arbitrary $D \neq 4$ the integrals $I_{0}(47)$ and $I_{1}$ (48) can be expressed only through infinite series. They belong to the class of integrals which was studied particulary in [17]. The first of them has already appeared in the calculation of the two-loop correction to the gluon Regge trajectory [18], where its limit at $\epsilon \rightarrow 0$ was found:

$$
I_{0}=\int \frac{d^{D-2} k_{1} d^{D-2} k_{2}}{\Lambda\left(\Gamma_{\epsilon}\right)^{2}} \frac{q^{2}}{k_{1}^{2}\left(k_{1}-q\right)^{2} k_{2}^{2}\left(k_{2}-q\right)^{2}} \ln \frac{q^{2}}{\left(k_{1}-k_{2}\right)^{2}}=\frac{1}{\epsilon}+15 \psi^{(2)}(1) \epsilon^{2}+\mathcal{O}\left(\epsilon^{3}\right) .
$$

Here $\Gamma_{\epsilon}$ and $\Lambda$ are given in (A6) and $(\mathrm{B} 1) ; \psi^{(2)}$ means the second derivative of $\psi$. We have obtained the limit of the second integral:

$$
I_{1}=\int \frac{d^{D-2} k_{1} d^{D-2} k_{2}}{\Lambda\left(\Gamma_{\epsilon}\right)^{2}} \frac{\left(k_{1}-k_{2}\right)^{2}}{k_{1}^{2}\left(k_{1}-q\right)^{2} k_{2}^{2}\left(k_{2}-q\right)^{2}} \ln \frac{q^{2}}{\left(k_{1}-k_{2}\right)^{2}}=-\frac{4}{\epsilon}+6 \psi^{(2)}(1) \epsilon^{2}+\mathcal{O}\left(\epsilon^{3}\right) .
$$

Below some details of the calculation are given.

Representing the integral as

$$
I_{1}=\left.\frac{d}{d \nu} I(\nu)\right|_{\nu=0}, I(\nu)=\int \frac{d^{D-2} k_{1} d^{D-2} k_{2}}{\Lambda\left(\Gamma_{\epsilon}\right)^{2}} \frac{\left(q^{2}\right)^{\nu}}{k_{1}^{2}\left(k_{1}-q\right)^{2} k_{2}^{2}\left(k_{2}-q\right)^{2}\left[\left(k_{1}-k_{2}\right)^{2}\right]^{\nu-1}}
$$

we have from Eqs. (1), (4), (9) of [17]:

$I(\nu)=\frac{2 \epsilon^{2}}{2 \epsilon-1} \frac{\nu^{-1}(\nu-\epsilon)}{\left(\Gamma_{\epsilon}\right)^{2}} G_{2}(1,1-\epsilon+\nu) G_{2}(1, \nu) \cdot\left(\nu(\nu-1) \lim _{b \rightarrow 0} S(\epsilon-1, b, 2 \epsilon-\nu, \nu-1-\epsilon)\right)$, 
where

$$
G_{2}\left(\alpha_{1}, \alpha_{2}\right)=G_{1}\left(\alpha_{1}\right) G_{1}\left(\alpha_{2}\right) G_{1}\left(2+2 \epsilon-\alpha_{1}-\alpha_{2}\right), G_{1}(\alpha)=\frac{\Gamma(1+\epsilon-\alpha)}{\Gamma(\alpha)}
$$

The function S(a,b,c,d) is defined by Eqs. (17), (16) and (10) of [17]:

$$
\left.S(a, b, c, d)=\frac{\pi \cot (\pi c)}{H(a, b, c, d)}-\frac{1}{c}-\frac{b+c}{b c} F(a+c,-b,-c, b+d)\right)
$$

where

$$
H(a, b, c, d)=\frac{\Gamma(1+a) \Gamma(1+b) \Gamma(1+c) \Gamma(1+d) \Gamma(1+a+b+c+d)}{\Gamma(1+a+c) \Gamma(1+a+d) \Gamma(1+b+c) \Gamma(1+b+d)},
$$

and $F(a, b, c, d)$ is expressed through the generalized hypergeometric function ${ }_{3} F_{2}$ :

$$
F(a, b, c, d)={ }_{3} F_{2}\left[\begin{array}{c}
-a,-b, 1 \\
1+c, 1+d
\end{array} ; 1\right]-1
$$

The limit $b \rightarrow 0$ in (C4) can be easily taken. We can use also for this purpose Eq.(12) of [17] and obtain with our values of parameters

$$
\nu(\nu-1) \lim _{b \rightarrow 0} S(\epsilon-1, b, 2 \epsilon-\nu, \nu-1-\epsilon)=S_{1}(\nu)+S_{2}(\nu)
$$

where

$$
\begin{gathered}
S_{1}(\nu)=\pi \cot [\pi(2 \epsilon-\nu)] \frac{\Gamma(3 \epsilon-\nu) \Gamma(1+\nu)}{\Gamma(\epsilon) \Gamma(2 \epsilon-1)}-\frac{\nu(\nu-1)}{2 \epsilon-\nu} ; \\
S_{2}(\nu)=\nu(\nu-1) \frac{3 \epsilon-1-\nu}{(\epsilon-1)(\nu-2 \epsilon)} F(1-\epsilon, 2 \epsilon-\nu, \nu-2 \epsilon, \nu-2) .
\end{gathered}
$$

With these denotations we have

$$
I_{1}=\left.\frac{d}{d \nu}\left(S_{0}(\nu)\left(S_{1}(\nu)+S_{2}(\nu)\right)\right)\right|_{\nu=0}
$$

where

$$
S_{0}(\nu)=\frac{\epsilon^{2}}{\left(\Gamma_{\epsilon}\right)^{2}} \frac{2}{2 \epsilon-1} \frac{\Gamma^{2}(\epsilon) \Gamma(2 \epsilon-\nu) \Gamma(1-2 \epsilon+\nu) \Gamma(1-\nu+\epsilon)}{\Gamma(3 \epsilon-\nu) \Gamma(1+\nu) \Gamma(1+2 \epsilon-\nu)} .
$$

We need to know $S_{i}(0)$ and $S_{i}^{\prime}(0), \quad i=0 \div 2$. For $i=0,1$ they are easily obtained from (C13), (C10):

$$
\begin{aligned}
& S_{0}(0)=\frac{3}{2 \epsilon-1} \mathrm{X}_{\Gamma}, \quad S_{0}^{\prime}(0)=S_{0}(0)\left(\frac{1}{6 \epsilon}+\Psi-\Psi_{2}\right) \\
& S_{1}(0)=2 \pi \cot [2 \pi \epsilon] \frac{\epsilon(2 \epsilon-1)}{3} \frac{\Gamma(1+3 \epsilon)}{\Gamma(1+\epsilon) \Gamma(1+2 \epsilon)} \\
& S_{1}^{\prime}(0)=S_{1}(0)\left(\psi(1)-\psi(1+3 \epsilon)+\frac{1}{3 \epsilon}+\frac{2 \pi}{\sin (4 \pi \epsilon)}\right)+\frac{1}{2 \epsilon}
\end{aligned}
$$


where $\mathrm{X}_{\Gamma}$ is defined in (A6), $\Psi$ and $\Psi_{2}$ in (49). To find $S_{2}(0)$ and $S_{2}^{\prime}(0)$ we use the integral representation

$$
F(a, b, c, d)=-1+\frac{\Gamma(1+d) \Gamma(1+c)}{\Gamma(d) \Gamma(-b) \Gamma(1+c+b)} \int_{0}^{1} d x(1-x)^{d-1} \int_{0}^{1} d z z^{-b-1}(1-z)^{c+b}(1-z x)^{a}
$$

which follows from the standard representation for the hypergeometric functions. Performing integration over $x$ by parts three times we obtain

$$
\begin{aligned}
S_{2}(\nu)=(3 \epsilon-1-\nu)\left(\frac{\nu}{1-2 \epsilon+\nu}\right. & +\frac{\epsilon}{2-2 \epsilon+\nu} \\
& \left.+\epsilon \int_{0}^{1} \int_{0}^{1} d x d z z^{1-2 \epsilon+\nu}(1-x)^{\nu} \frac{d}{d x}(1-z x)^{-1-\epsilon}\right),
\end{aligned}
$$

so that

$$
\begin{gathered}
S_{2}(0)=(1-2 \epsilon) \frac{\Gamma(1-\epsilon) \Gamma(1-2 \epsilon)}{\Gamma(1-3 \epsilon)}, \\
S_{2}^{\prime}(0)=\frac{S_{2}(0)}{1-3 \epsilon}+(3 \epsilon-1)\left(\frac{1}{1-2 \epsilon}-\frac{\epsilon}{4(1-\epsilon)^{2}}+\epsilon\left(J_{1}+J_{2}\right)\right),
\end{gathered}
$$

where

$$
\begin{aligned}
& J_{1}=\int_{0}^{1} \int_{0}^{1} d x d z z^{1-2 \epsilon} \ln z \frac{d}{d x}(1-z x)^{-1-\epsilon}, \\
& J_{2}=\int_{0}^{1} \int_{0}^{1} d z d x z^{1-2 \epsilon} \ln (1-x) \frac{d}{d x}(1-z x)^{-1-\epsilon} .
\end{aligned}
$$

The integral $J_{1}$ can be easily found:

$$
J_{1}=\frac{1}{4(1-\epsilon)^{2}}+\frac{\Gamma(1-\epsilon) \Gamma(2-2 \epsilon)}{\Gamma(2-3 \epsilon)} \frac{(\psi(2-3 \epsilon)-\psi(2-2 \epsilon))}{\epsilon} .
$$

For the integral $J_{2}$, replacing $d / d x$ by $(z / x) d / d z$ in the representation $(\mathrm{C} 19)$ and integrating over $z$ by parts we obtain in the limit $\epsilon \rightarrow 0$

$$
J_{2} \simeq \frac{-1}{\epsilon^{2}}+1+\psi^{(1)}(1)+\epsilon\left(5-\psi^{(1)}(1)+\frac{\psi^{(2)}(1)}{2}\right)
$$

Using in (C12) the results $(\mathrm{C} 20),(\mathrm{C} 21),(\mathrm{C} 14)$ and $(\mathrm{C} 17)$ we get the final result (C2).

1. L. V. Gribov, E. M. Levin and M. G. Ryskin, Phys. Rep. C100 1 (1983).

2. G. Altarelli, Phys. Rep. 811 (1982). 
3. M. T. Grisaru, H. J. Schnitzer and H.-S. Tsao, Phys. Rev. Lett. 30811 (1973); Phys. Rev. D 84498 (1973).

4. L. N. Lipatov, Yad. Fiz. 23642 (1976) [Sov. J. Nucl. Phys. 23338 (1976)].

5. V. S. Fadin, E. A. Kuraev and L. N. Lipatov, Phys. Lett. B 6050 (1975); L. N. Lipatov, E. A. Kuraev and V. S. Fadin, and Zh. Éksp. Teor. Fiz. 71840 (1976) [Sov. Phys. JETP 44 $443(1976)]$.

6. V. S. Fadin and V. E. Sherman, Pis'ma Zh. Éksp. Teor. Fiz. 23599 (1976) [Sov. Phys. JETP Lett. 23548 (1976)]; Zh. Éksp. Teor. Fiz. 721640 (1977) [Sov. Phys. JETP 45861 (1977)].

7. M. Gell-Man, M. L. Goldberger, F. E. Low, E. Marx and F. Zachariasen, Phys. Rev. B133 145 (1964); B. M. McCoy and T. T. Wu, Phys. Rev. D13 369 (1976).

8. S. Mandelstam, Phys. Rev. B137 949 (1965).

9. E. A. Kuraev, L. N. Lipatov and V. S. Fadin, Zh. Éksp. Teor. Fiz. 72377 (1977) [Sov. Phys. JETP 45199 (1977)]; Ya. Ya. Balitskii and L. N. Lipatov, Sov. J. Nucl. Phys. 28822 (1978).

10. V. S. Fadin, Talk at NATO Advanced Research Workshop on Diffraction 2002, Alushta, Ukraine, 31 Aug - 6 Sep 2002, in Alushta 2002, Diffraction 2002, pp. 235-245.

11. Ya. Ya. Balitskii, L. N. Lipatov and V. S. Fadin, in Proceedings of XIV LIYAF Winter School (Leningrad, 1979), p. 109.

12. V. S. Fadin, Yad. Fiz. 662067 (2003); Phys. Atom. Nucl. 66 (2003) 2017.

13. L. N. Lipatov, M. I. Vyasovsky, Nucl. Phys. B597 399 (2001), arXiv:hep-ph/0009340.

14. V. S. Fadin and R. Fiore, Phys. Rev. D64 114012 (2001), arXiv:hep-ph/0107010.

15. M. I. Kotsky, L. N. Lipatov, A. Principe and M. I. Vyazovsky, Nucl. Phys. B648 277 (2003), arXiv:hep-ph/0207169.

16. A. V. Bogdan, V. Del Duca, V. S. Fadin and E. W. N. Glover, JHEP 20332 (2002), arXiv:hep$\mathrm{ph} / 0201240$.

17. D. J. Broadhurst, J. A. Gracey and D. Kreimer, Z. Phys. C75 559 (1997), arXiv:hepth/9607174.

18. V. S. Fadin, R. Fiore and M. I. Kotsky, Phys. Lett. B387 593 (1996), arXiv:hep-ph/9605357. 


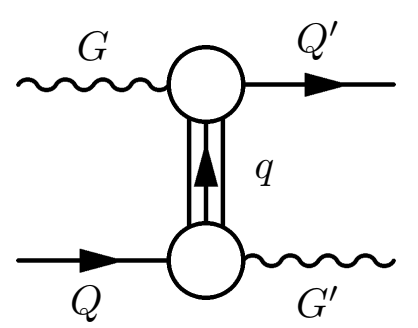

Figure 1. Schematic representation of the backward quark-gluon scattering process $G+Q \rightarrow Q^{\prime}+G^{\prime}$. The triple line denotes an intermediate $t$-channel state with momentum $q=p_{Q}-p_{G^{\prime}}=p_{Q^{\prime}}-p_{G}$.
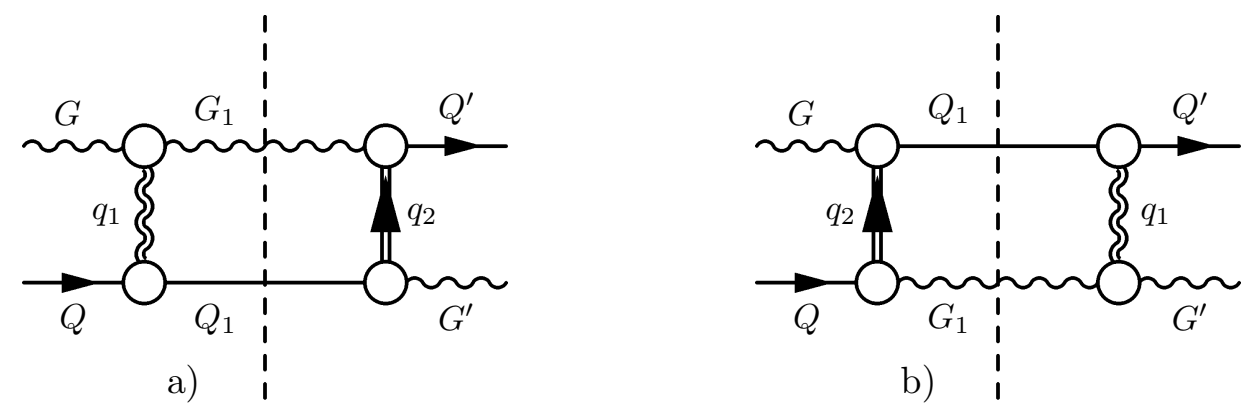

Figure 2. Schematic representation of the two-particle contribution to the $s$-channel discontinuity of the backward quark-gluon scattering amplitude. The doubled lines represent Reggeized quark and gluon, and the blobs - PPR vertices.
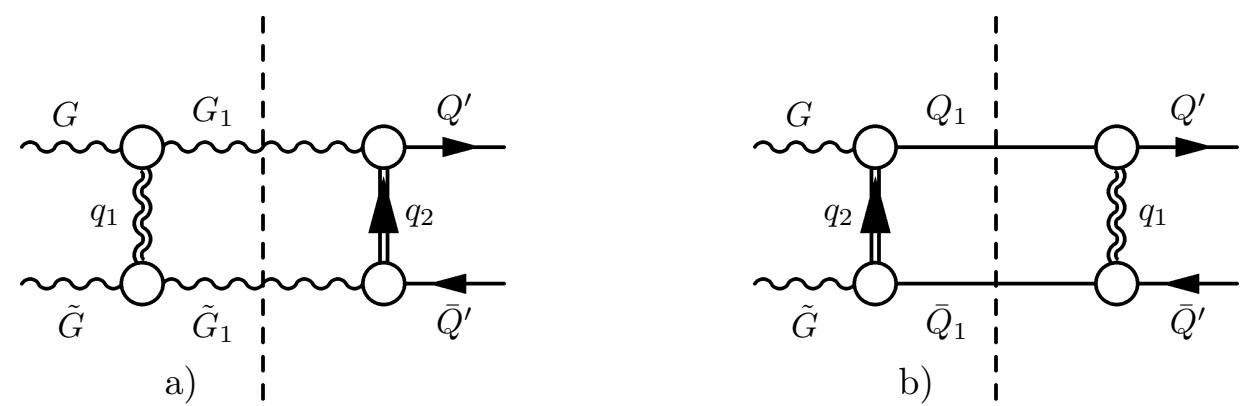

Figure 3. Schematic representation of the two-particle contribution to the cross channel discontinuity of the backward quark-gluon scattering amplitude. 